OPEN ACCESS

Edited by:

Adi Avni,

Tel Aviv University, Israel

Reviewed by:

Bala Rathinasabapathi,

University of Florida, USA

Zonghua Wang,

Fujian Agriculture and Forestry

University, China

*Correspondence:

Hongmei Zeng

zenghongmei@caas.cn

Specialty section:

This article was submitted to

Plant Biotic Interactions,

a section of the journal

Frontiers in Plant Science

Received: 30 May 2016 Accepted: 18 November 2016 Published: 01 December 2016

Citation:

Lv S, Wang Z, Yang X, Guo L, Qiu D and Zeng $H$ (2016) Transcriptional Profiling of Rice Treated with MoHrip1 Reveal the Function of Protein Elicitor in Enhancement of Disease Resistance and Plant Growth.

Front. Plant Sci. 7:1818. doi: $10.3389 /$ fpls.2016.01818

\section{Transcriptional Profiling of Rice Treated with MoHrip1 Reveal the Function of Protein Elicitor in Enhancement of Disease Resistance and Plant Growth}

\author{
Shun Lv, Zhenzhen Wang, Xiufen Yang, Lihua Guo, Dewen Qiu and Hongmei Zeng * \\ State Key Laboratory for Biology of Plant Diseases and Insect Pests, Institute of Plant Protection, Chinese Academy of \\ Agricultural Sciences, Beijing, China
}

MoHrip1 is a protein elicitor isolated from Magnaporthe oryzae and was found to induce blast-resistance in rice. To investigate the comprehensive functions of MoHrip1, next-generation sequencing (NGS)-based digital gene expression (DGE) profiling was performed to collect the transcriptional data of differentially expressed genes (DEGs) induced by MoHrip1. A total of 308 genes were identified with differential expression, and 80 genes were predicted to be induced specifically by MoHrip1. Among these 308 genes, a series of genes associated with the salicylic acid (SA) pathway, phytoalexin, transcription factors, and pathogen-related proteins were identified. Both the SA signaling pathway and the gibberellin (GA) pathway were activated, while the jasmonic acid (JA) signaling pathway was repressed. The contents of endogenous SA and GA and the morphological characteristics of the rice after treatment were measured to provide evidence supporting the predictions made based on the DGE data. The 80 genes mentioned above might be candidate genes for studying interactions with MoHrip1. The transcriptional data provided global effect information in rice induced by MoHrip1, and all the results demonstrated that MoHrip1 could induce pathogen resistance and promote plant growth by regulating the contents of SA and GA directly or indirectly.

Keywords: elicitor, MoHrip1, DGE (digital gene expression profiling) sequencing, disease-resistance, signaling pathways

\section{INTRODUCTION}

Rice blast, a major fungal disease caused by Magnaporthe oryzae, seriously affects rice production worldwide (Ribot et al., 2008), leading to yield losses of 50-70\% (Bagnaresi et al., 2012). As rice is the world's second most important human food crop (FAOSTAT, 2014), it is necessary to develop methods to protect it from pathogenic diseases and to increase its production. Increasing research on the molecular events governing the interactions between rice and M. oryzae have contributed to methods for improving disease resistance (Chen and Ronald, 2011).

The elicitors include molecules isolated from various organisms, including viruses, oomycetes, bacteria, and fungi. Some elicitors have been used to improve the pathogen resistance of plants (Bent and Mackey, 2007). Such elicitor molecules include oligosaccharides, lipids, peptides, glycoproteins, and proteins (Nürnberger, 1999; De Wit et al., 2009; Ellis et al., 2009). 
A range of elicitors was isolated from M. oryzae, including sphingolipids, proteins, and glycoproteins. These elicitors induce resistance responses and hypersensitive cell death in rice, while inducing systemic acquired resistance (SAR) in plants (Koga et al., 1998; Qiu et al., 2009; Peng et al., 2011). However, these elicitor-activity compounds were not secretory proteins. During the whole genome sequencing and research on the $M$. oryzae proteome, several secreted proteins were identified and characterized, such as MC69 (Saitoh et al., 2012), SLP1 (Mentlak et al., 2012), Avr-effectors (PWL1, PWL2, AvrPi-ta, AvrPiz-t, Avr-Pia, AvrPii, Avr-Pik/km/kp, Avr1-CO39, ACE1) (Liu et al., 2013), and MoCDIP1-5 (Chen et al., 2013). MoHrip1 is a secreted protein elicitor isolated from $M$. oryzae and can enhance the defense of rice seedlings against infection by $M$. oryzae (Chen et al., 2012).

Next-generation sequencing (NGS) technologies are rapidly evolving and are changing biology research (Metzker, 2010). Digital gene expression (DGE) is a relatively new approach based on NGS technology for the study of the transcriptome, and this method has advantages in studying genes that are expressed differentially (Tao et al., 2012). The sequencing of the Rice cv. Nipponbare (Oryza sativa spp. japonica) genome has been implemented and published (Sasaki and Burr, 2000; Kawahara et al., 2013), making sequencing-based transcriptome studies (i.e., DGE) more accessible. To our knowledge, there have been some RNA-seq studies focusing on rice resistance genes and the interactions between pathogens and rice (Bagnaresi et al., 2012). Furthermore, several studies aiming to identify differences in the gene expression in rice suspension cells treated with elicitors have been completed (Kim et al., 2000). However, no NGS-based transcriptome studies have been conducted focusing on genes induced by the mutual effects of intact rice and pathogen-derived elicitors.

In this research, we employed DGE-based RNA-seq to study the gene expression in rice induced by MoHrip1. Comparisons between the treatment and controls allowed us to identify genes sensitive to the elicitors. Many differentially expressed genes (DEGs) related to the defense response were identified, along with genes associated with plant growth. Even some specific genes that were not expressed in untreated rice but that were upregulated in rice after treatment were identified. Our results may widen the functional scope of the elicitor in inducing resistance and may help identify genes that interact with the elicitor.

\section{METHODS}

\section{Plant Material and Elicitor Preparation}

Rice cv. Nipponbare (O. sativa spp. japonica) was used in this study. For RNA-seq, the rice seeds were sterilized by immersion in $2 \%$ sodium hypochlorite for $30 \mathrm{~min}$. After they were washed with deionized water 7-8 times, the seeds were germinated on $1 / 2$ Murashige and Skoog (MS) medium for 5 days and transferred to nutritional soil (Park et al., 2012). Rice plants were cultured in a growth chamber at $30^{\circ} \mathrm{C}$ in light and $25^{\circ} \mathrm{C}$ in dark, with a 12-h light/dark photoperiod. We treated the rice seedlings by spraying with either elicitor $(30 \mu \mathrm{M})$ or Tris- $\mathrm{Cl}(25 \mathrm{mM})$ as a negative control at the three-leaf stage. Both the treated rice and the negative control were sampled in two replicates at 0,24 , $48,72 \mathrm{~h}$ after treatment (hat), separately. Samples for RNA-seq were collected and frozen in liquid nitrogen and stored at $-80^{\circ} \mathrm{C}$ until use.

The MoHrip1 used in this study was expressed and purified as previously described (Chen et al., 2012; Zhang et al., 2013). The recombinant expression vector containing the MoHrip1 gene was transformed into E. coli strain transetta (DE3) competent cells (TransGen Biotech, Beijing, China) to express the elicitor. The bacteria were first grown to achieve an $\mathrm{OD}_{600}$ of 0.8 in Luria Bertani (LB) medium at $37^{\circ} \mathrm{C}$. Then, the protein was induced upon the addition of $0.1 \mathrm{mM}$ isopropyl $\beta$-D-1-thiogalactopyranoside (Sigma, St. Louis, MO, USA) at $16^{\circ} \mathrm{C}$. Twelve hours after induction, the cells were collected by centrifugation and resuspension. After ultrasonication and centrifugation, the supernatant containing the recombinant protein was obtained. The protein purification mainly consisted of two procedures: affinity chromatography with a His-Trap HP column (GE Healthcare, Waukesha, WI, USA) and ion-exchange chromatography with a Mono Q column (GE Healthcare, Waukesha, WI, USA). After concentration, the purified protein was detected by SDS-PAGE and was quantified using the $\mathrm{BCA}^{\mathrm{TM}}$ Protein Assay Kit (Pierce, Rockford, IL, USA).

\section{DGE Library Preparation and Sequencing}

A total of 14 samples (two replicates of non-treated rice and two replicates of treated and control rice collected at three time points) were prepared for RNA extraction. The total RNA was isolated using an RNAprep Plant Kit (TIANGEN Biotech, Beijing, China) according to the manufacturer's protocol. RNA purity was checked using a NanoPhotometer ${ }^{\circledR}$ spectrophotometer (IMPLEN, CA, USA). RNA integrity was confirmed using an RNA Nano 6000 Assay Kit for the Agilent Bioanalyzer 2100 system (Agilent Technologies, CA, USA) with a minimum RNA integrated number (RIN) value of 7 .

A total of $3 \mu \mathrm{g}$ of RNA per sample was used as the input material for the RNA sample preparations. Sequencing libraries were generated using the NEBNext ${ }^{\circledR}$ Ultra ${ }^{\mathrm{TM}}$ RNA Library Prep Kit for Illumina ${ }^{\circledR}$ (NEB, USA) following the manufacturer's recommendations. In brief, mRNA was purified from the total RNA using oligo (dT) magnetic beads. After fragmentation with divalent cations in NEBNext First Strand Synthesis Reaction Buffer (5X), first strand cDNA was synthesized using random hexamer primers and M-MuLV Reverse Transcriptase (RNase $\mathrm{H}^{-}$). Then, second strand cDNA synthesis was performed using DNA Polymerase I and RNase H. To select cDNA fragments $150-$ $200 \mathrm{bp}$ in length, the library fragments were purified using the AMPure XP system (Beckman Coulter, Beverly, USA). PCR was then performed with Phusion High-Fidelity DNA polymerase, Universal PCR primers and an Index (X) Primer. Finally, the PCR products were purified (AMPure XP system), and the library quality was assessed on an Agilent Bioanalyzer 2100 system.

The clustering of the samples was performed on a cBot Cluster Generation System using a TruSeq PE Cluster Kit v3cBot-HS (Illumina) according to the manufacturer's instructions. After cluster generation, the library was sequenced on an 
Illumina HiSeq 2000 platform, and 100-bp paired-end reads were generated.

\section{RNA-Seq Read Mapping and DE Gene Clustering}

The sequence data sets are available at the NCBI Short Read Archive (SRA) under the accession number SRA325858. Raw data had adaptor fragments and a few low-quality sequences, along with several types of impurities. Clean data were obtained by removing low-quality reads containing adapters or poly- $\mathrm{N}$ sequences from the raw data. All downstream analyses were based on clean, high-quality data.

The reference genome and gene model annotation files were downloaded from the genome website directly. The reference genome index was built using Bowtie v2.2.3 (Langmead et al., 2009), and paired-end clean reads were aligned to the reference genome using TopHat v2.0.12 (Trapnell et al., 2009). TopHat was selected as the mapping tool, as it has the advantage of being able to generate a database of splice junctions based on the gene model annotation file and, thus, to produce better mapping results than other non-splice mapping tools. HTSeq v0.6.1 (Anders, 2010) was used to count the read numbers mapped to each gene. The reads per kilobase of transcript per million mapped reads (RPKM) of each gene was calculated based on the length of the gene and the read counts mapped to that gene (Mortazavi et al., 2008).

\section{Analysis of Differentially Expressed Genes}

The DESeq R package (1.10.1) (Wang et al., 2010) was introduced to perform differential expression analysis of two conditions (two biological replicates per condition). DESeq (Anders and Huber, 2010) provides statistical methods for identifying differential expression in DGE data using a model based on the negative binomial distribution. The resulting $P$-values were adjusted using the Benjamini and Hochberg's approach for controlling the false discovery rate (FDR) (Benjamini and Hochberg, 1995). Genes with a FDR adjusted $P<0.05$, as found by DESeq, were defined as being significant differentially expressed.

Gene Ontology (GO) enrichment analysis of the DEGs was implemented in the GOseq (Young et al., 2010) R package, in which the gene length bias was corrected. GO terms with corrected $P<0.05$ were considered significantly enriched in the DEGs. KEGG (Kyoto Encyclopedia of Genes and Genomes) (Kanehisa et al., 2008) is a database resource for understanding the high-level functions and utilities of a biological system. KEGG has abundant molecular-level information, especially in the format of large-scale molecular datasets generated by genome sequencing and other high-throughput experimental technologies. KOBAS (Mao et al., 2005) software was used to test the statistical enrichment of the DEGs in the KEGG pathway.

\section{Real-Time Quantitative RT-PCR Assay}

For each condition, three independent RNA samples were used to validate gene expression level by performing quantitative realtime PCR. The cDNAs used as the template of Quantitative RT-PCR were produced using the TransScript All-in-One FirstStrand cDNA Synthesis SuperMix for qPCR (TransGen Biotech,
Beijing, China), and the concentrations of the mRNAs were adjusted to be the same. Quantitative RT-PCR was performed using SuperReal PreMix Plus (TIANGEN Biotech, Beijing, China). Each real-time RT-PCR reaction $(20 \mu \mathrm{L})$ included 25 ng cDNA, $0.3 \mu \mathrm{M}$ of each primer, and $1 \times$ SYBR Green PreMix. All reactions were performed in three technical replicates on a Bio-rad CFX96 Real-Time PCR Detection System (Bio-Rad, CA, USA) under the following conditions: $95^{\circ} \mathrm{C}$ for $15 \mathrm{~min}$ and 40 cycles of $95^{\circ} \mathrm{C}$ for $10 \mathrm{~s}, 55^{\circ} \mathrm{C}$ for $30 \mathrm{~s}$, and $72^{\circ} \mathrm{C}$ for $32 \mathrm{~s}$ to determine the fluorescence level and to calculate cycle threshold (Ct) values, followed by increments of $0.5^{\circ} \mathrm{C}$ for $5 \mathrm{~s}$ along a gradient from $65^{\circ} \mathrm{C}$ to $95^{\circ} \mathrm{C}$. Melting curves were obtained to ensure primer specificity. Gene relative expression level was obtained by comparing with non-treated rice after normalization with reference gene using the $2^{-\Delta \Delta C t}$ approach. Osactin was used as the reference gene, and the gene IDs and primer sequences are listed in Table S1. Data were statistically validated by a correlation test using the Pearson's method.

\section{Determination of the Endogenous Levels of Salicylic Acid (SA)}

For the SA content assay, the rice cultivation method was the same as was used for the RNA-seq. Treated and control rice samples were collected in three replicates at $0,12,24$, 36, 48, 60, $72 \mathrm{~h}$ after treatment (hat). Leaf tissue treated with MoHrip1 or buffer was collected, weighed and frozen in liquid nitrogen. For each sample, $0.1 \mathrm{~g}$ of the frozen tissue was extracted and quantitated for free SA, as described previously (Bowling et al., 1994). In brief, the tissue was ground into powder and homogenized in $1 \mathrm{~mL}$ of methanol- $\mathrm{H}_{2} \mathrm{O}$-acetic acid (80:19:1). After extraction overnight at $4^{\circ} \mathrm{C}$ and centrifugation, the supernatant was re-extracted with the solution described previously. After the addition of $1 \mathrm{~mL}$ chloroform and further centrifugation, the organic phase containing the free SA was dried in a speed vacuum with heat $\left(\sim 40^{\circ} \mathrm{C}\right)$. The residue was resuspended in $0.5 \mathrm{~mL}$ of methanol, filtered and analyzed by Ultra Performance Liquid Chromatography (UPLC). UPLC was performed on an ACQUITY UPLC@BEHC 18 column $(50 \mathrm{~mm}$ $\times 2.1 \mathrm{~mm}, 1.7 \mu \mathrm{m})$ run at $40^{\circ} \mathrm{C}$ with a flow rate of $0.4 \mathrm{~mL}$ $\mathrm{min}^{-1}$. The analytes were eluted from the column with a mixed solvent of water with $0.1 \%$ acetic acid (solvent $\mathrm{A}$ ) and methanol with $0.1 \%$ acetic acid (solvent $\mathrm{B}$ ) using a linear gradient mode (Matsuura et al., 2009). The ratio of A and B was 90:10 from $0 \mathrm{~s}$ to $3 \mathrm{~min}$, and this ratio changed linearly from 90:10 to 10:90 between 3 and $4 \mathrm{~min}$. The ratio of 90:10 was finally maintained from 4 to $7 \mathrm{~min}$. The authenticity of the SA from rice leaf extract was verified based on the retention times and spectral properties, which matched perfectly to those of commercial SA standards.

\section{Growth Promotion Assay and Determination of Endogenous Levels of GA} For the GA assay, the rice seeds were sterilized by immersion in $75 \%$ ethanol for $5 \mathrm{~min}$. After they were washed with sterile water, seeds were immersed in different concentrations of elicitor dilution $(2.5,5,10$, and $20 \mu \mathrm{g} / \mathrm{mL})$ or $\mathrm{H}_{2} \mathrm{O}$ as a negative control for $8 \mathrm{~h}$. Then, the germinated seeds were transferred to liquid 
MS medium for cultivation. The leaf tissue was collected at 9, 11, and 13 days after the rice seedlings were transferred to liquid MS medium with three replicates. For the growth promotion assay, the lengths of the seedlings and roots were measured, as were the weights of the whole plants. To assess the endogenous GA levels, the leaf tissue was ground into powder in liquid nitrogen after the weight measurement and addition of phosphate buffer $(\mathrm{pH} 7.4$, $0.01 \mathrm{~mol} / \mathrm{L}$ ), with a ratio of 1:9. The supernatant was collected after centrifugation. Then, the GA level was determined using a Plant Gibberellic Acid ELISA Kit (FUYING Biotech, Shanghai, China) following the solid-phase enzyme immunoassay method (Atzorn and Weiler, 1983). In brief, the supernatant was added to a microtiter plate coated well with purified plant GA antibody and was then incubated at $37^{\circ} \mathrm{C}$. The chromogen solution was then added following the addition of horseradish peroxidase (HRP)-conjugate reagent and incubated at $37^{\circ} \mathrm{C}$. After adding the stop solution, the absorbance was obtained at $450 \mathrm{~nm}$ and was matched with the GA standard curve to calculate the endogenous GA levels of the leaf tissues.

\section{Bioassay for MoHrip1-Induced Disease Resistance in Rice}

For the disease resistance assay, the rice seeds were divided into three groups. Each group contains 25 rice seeds with three replicates. The MM group indicated that the rice seeds were immersed in MoHrip1 $(10 \mu \mathrm{g} / \mathrm{mL})$ as the GA assay then sprayed with MoHrip1 $(30 \mu \mathrm{M})$ at three-leaf stage as assay for RNAseq mentioned above. The $\mathrm{M}$ group indicated that the rice seeds were immersed in MoHrip1 $(10 \mu \mathrm{g} / \mathrm{mL})$ then sprayed with Tris$\mathrm{Cl}(25 \mathrm{mM})$ at three-leaf stage. The CK group indicated that the rice seeds were immersed in $\mathrm{H}_{2} \mathrm{O}$ then sprayed with Tris$\mathrm{Cl}(25 \mathrm{mM})$ at three-leaf stage as a negative control. After 3 days of incubation, three groups of rice plants were sprayed with an aqueous suspension of $1 \times 106 \mathrm{M}$. oryzae $(\mathrm{KJ} 201)$ spores per milliliter containing $0.05 \%(\mathrm{v} / \mathrm{v})$ Tween 20 . The inoculated rice plants were maintained at $26^{\circ} \mathrm{C}$ and $100 \%$ relative humidity in a dark chamber for $24 \mathrm{~h}$. Then we modified the growth chamber with $70-80 \%$ relative humidity under a 14 -h-light/10h-dark photoperiod (Chen et al., 2012). Leaf blast symptoms were investigated at 7 days post-inoculation when typical lesions appeared on the leaves of negative control rice plants. The disease indices of plants inoculated with rice blast were compared. Each seedling was researched and rated on a scale of $0-9(0=$ resistant and $9=$ susceptible) according to the international specification for rice blast disease.

\section{RESULTS}

\section{DGE Sequencing of the MoHrip1-Treated Rice}

A previous study confirmed that rice treated with MoHrip1 show enhanced resistance to M. oryzae (Chen et al., 2012). To obtain the global gene expression profile differences of rice treated with MoHrip1 compared to those treated with buffer (Tris-Cl) as a negative control, 24, 48, and 72 hat and non-treated rice samples were prepared and sequenced on the
Illumina sequencing platform. A total of 14 DGE libraries were constructed to identify differences in the gene expression levels. The 14 libraries included non-treated rice samples (designated as NoT0), rice samples of MoHrip1 and buffer at 24 hat (designated as MoT1 and BuT1), rice samples of MoHrip1 and buffer at 48 hat (designated as MoT2 and BuT2), and rice samples of MoHrip1 and buffer at 72 hat (designated as MoT3 and BuT3), with two replicates each. After removing reads containing adapters, reads containing poly-N sequences, and low-quality reads, the total clean reads per library ranged from 10.3 to 14.8 million. The total reads mapped to the Rice $\mathrm{cv}$. Nipponbare genome according to the TopHat analysis ranged from 9.5 to 14.4 million (Table 1). RPKM was introduced to calculate the expression level of each gene. The value of "RPKM $\geq 1$ or $<1$ " was used as the threshold to identify whether a gene was expressed or not (Table 2).

A saturation curve was constructed to determine whether the amount of data fulfilled the requirement of quantifying the gene expression level. The higher the gene expression level, the easier it was to accurately quantify the gene (Figure S1). Biological replicates were necessary for high-throughput sequencing to obtain reliable analysis results (Hansen et al., 2011). Our experiments showed extremely high levels of correlation for each pair of biological replicates [in all cases examined, the Pearson correlation coefficient $(\mathrm{PCC})>0.97$; Figure S2], indicating that our sampling, assay, and analysis methods were steady and robust.

GO enrichment analysis was performed to classify the gene functions of the detected DEGs. Based on the sequencing data, the genes detected differentially in the treated rice after 1 day were enriched in two domains: biological process and molecular function. The cardinal enriched terms were metabolic and oxidation-reduction processes in the biological process domain and oxidoreductase lyase activity in molecular function. Genes detected differentially in treated rice after 2 days were enriched in only one domain: the biological process domain, and the cardinal term was metabolic process. In contrast, the genes detected differentially in the treated rice after 3 days were not found to be enriched (Figure S3).

To characterize the complex biological behaviors for the transcriptome, the KEGG database was used to analyze the pathway annotations for the DEGs. In the three rice groups being compared between the treatment and control conditions, only genes in the rice treated for 1 day showed significant pathway enrichment. The pathways with the most representation were the phenylalanine, tyrosine, tryptophan, diterpenoid, phenylpropanoid, amino acid, and secondary metabolite biosynthesis pathways and the plant-pathogen interaction pathways (Figure S4; Table S2). These annotations provided clues for investigating specific processes, especially those involved in the biosynthesis of secondary metabolites and plant-pathogen interactions.

Nineteen genes with a range of expression levels were selected for the validation of the gene expression levels estimated by RPKM using quantitative RT-PCR (qRT-PCR). The expression levels of the 19 genes determined by the qRT-PCR analysis using 7 different RNA samples generated 133 data points. The data for the cycle threshold (Ct) value and the $\log _{2}$ RPKM value of 


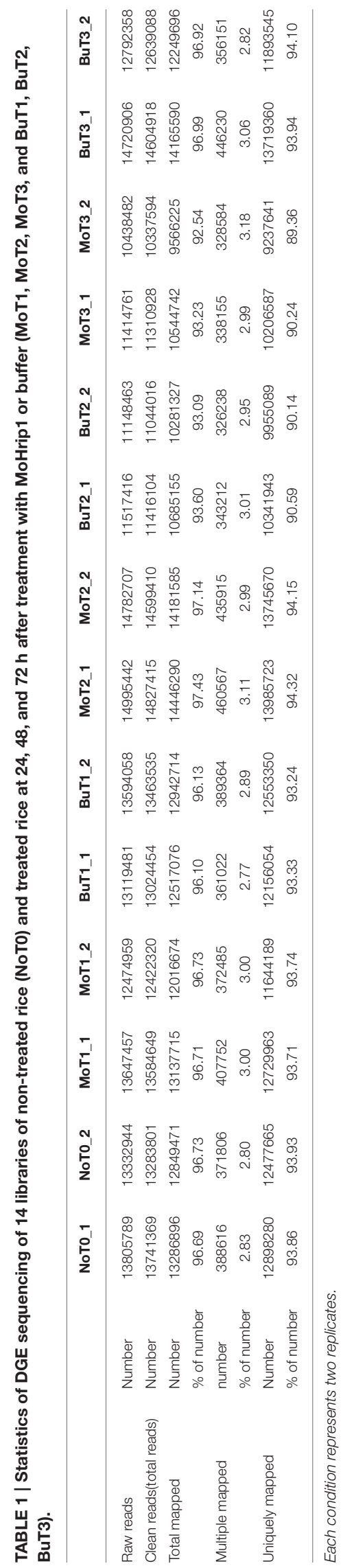

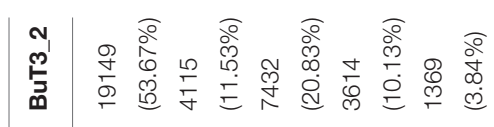

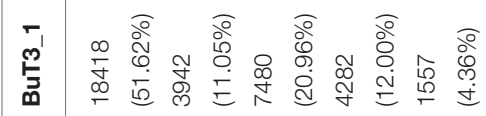

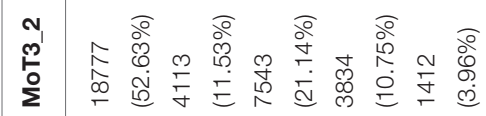

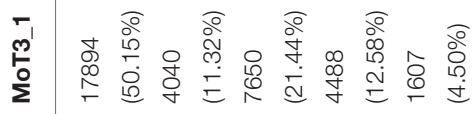

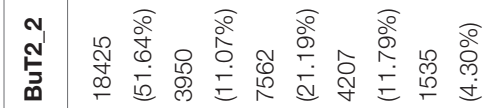

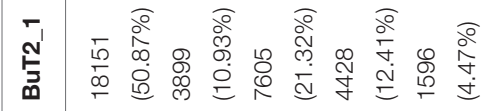

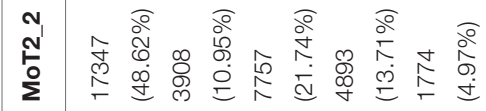

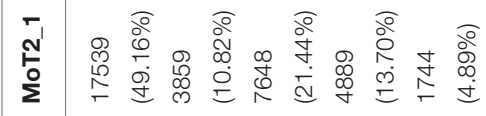

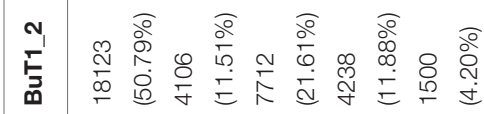

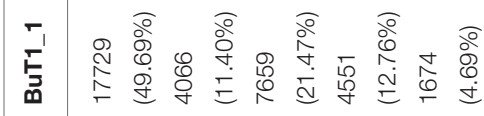

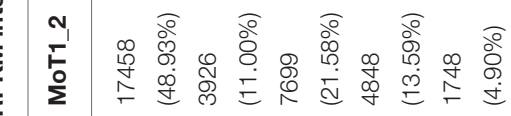

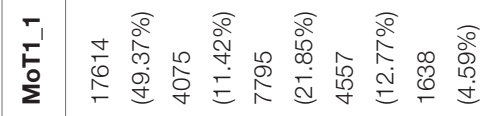

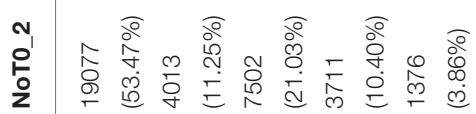

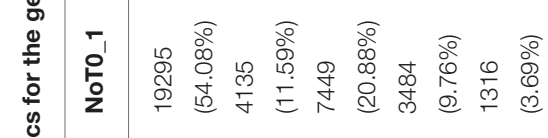

站 


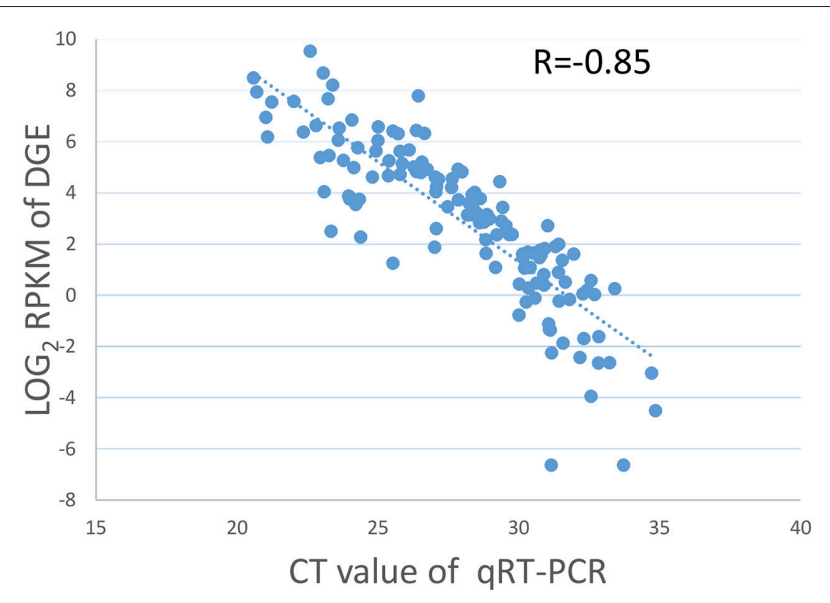

FIGURE 1 | Validation of DGE data by qRT-PCR. Scatter plots indicate the Ct results of the qRT-PCR and the $\log _{2}$ RPKM values of DGE for 133 data points from 19 genes in 7 different samples with a Pearson correlation coefficient of $R=-0.85$.

the selected genes are given in Table S3. The CT value of the qRT-PCR results correlated well with the $\log _{2}$ RPKM values of the DGE, with a PCC of $R=-0.85$ (Figure 1), indicating that the gene expression levels determined by DGE analysis were positively correlated with those determined by qRT-PCR.

\section{Differential Gene Expression Analysis}

To identify the genes induced by treatment with MoHrip1 and to assess gene expression pattern changes, we next evaluated the DEGs at all-time points. The final RPKM of one gene with replicates in the same condition was the average value for all replicate data. In general, the absolute value of " $\mid \log _{2}$ (FoldChange) $\mid>1$ " and the " $q<0.005$ " were used as the threshold to identify the DEGs in a sequencing experiment without replicates. Now that DESeq was used to eliminate biological deviation in the study with replicates, we set padj $<0.05$ as filtering standard for genes that were expressed differentially.

The generated libraries formed 9 volcano plots and 3 Venn diagrams based on 9 DEG comparisons (MoT1 vs. NoT0, MoT2 vs. NoT0, MoT3 vs. NoT0, BuT1 vs. NoT0, BuT2 vs. NoT0, BuT3 vs. NoT0, MoT1 vs. BuT1, MoT2 vs. BuT2, and MoT3 vs. BuT3). Based on the volcano plot, 214 DEGs were obtained at 24 hat, including 163 up-regulated genes and 51 down-regulated genes. Furthermore, 103 DEGs were obtained at 48 hat, including 78 upregulated genes and 25 down-regulated genes. Finally, 21 DEGs were obtained at 72 hat, including 14 up-regulated genes and 7 down-regulated genes (Figure S5). A Venn diagram was used to obtain the overlapping DEGs in the comparison group to see which DGEs were induced transiently and which DGEs were induced over a relatively long period of time. In this experiment, 28 genes were induced at both 24 and 48 h, 1 gene was induced at both 24 and $72 \mathrm{~h}$, and 1 gene was induced at both 48 and $72 \mathrm{~h}$, indicating that these genes may play important roles in induced resistance after treatment with MoHrip1 (Figure S6A).
It can also be concluded through the volcano plot and Venn diagram that both the treatment and the control conditions could induce gene expression differentially compared with the nontreated condition, while the treated samples induced more DGEs relative to the non-treated condition than the control samples (Figures S6B,C, S7).

\section{Differences in Gene Expression Patterns As Assessed by Clustering Analysis}

A total of 308 DEGs ( $\mid \log _{2}$ (FoldChange) $\mid>1, q<0.005$ ) were identified when rice samples treated with MoHrip1 or buffer after 24, 48, $72 \mathrm{~h}$ were compared with non-treated rice samples. All genes were used to generate clusters using the clustering affinity search technique (CAST). We identified two representative hierarchical clusters, consisting of a total of 24 genes, showing interesting regulation patterns at 24 and 48 hat (Figure 2). In the MoHrip1-treated group, these 24 genes were induced at 24 and 48 hat; in the buffer-treated group, these 24 genes were suppressed at all-time intervals that we analyzed (24, 48, 72 hat). These genes included peroxidase N (prxRPN), the $\mathrm{ABC}$ transporter (PDR20), and the heat stress transcription factor (Spl7). Two genes were specifically associated with the shikimate pathway (DHQDT/SDH, EPSPS) and three genes were specifically associated with the tryptophan pathway (IGPS, PAT, TS; Table S4) (Maeda and Dudareva, 2012). These shikimate and tryptophan pathway-associated genes were also included in the KEGG enrichment (data not shown). These results suggest that MoHrip1 can induce genes in several ways in rice, while the buffer has no such effect.

\section{DGE Analysis Reveals the Molecular Events Induced by MoHrip1 in Rice}

Previous studies have identified genes and pathways that participate in or are related to the resistance of blast. In our DGE data, we examined how these genes were expressed in rice after treatment with MoHrip1. This research aimed to identify genes or pathways critical for blast-resistance in rice, especially those induced by MoHrip1.

\section{Pathogenesis-Related Proteins}

The importance of pathogenesis-related proteins (PRs) in blast-resistance has been reported (Agrawal et al., 2001; Muthukrishnan et al., 2001; Hwang et al., 2007). Among the 35,679 genes detected in at least one of the 14 DGE libraries, 13 genes encode PR proteins, including 2 copies of pathogenesisrelated protein 1 (PR1), $6 \beta$-1-3-glucanases (PR2), 1 chitinase (PR3), 2 thaumatin-like proteins (PR5), and 2 prebenazoleinduced genes (PR10). Among the 2 PR1 genes, increased transcripts accumulated in the treated samples in one gene (OS07G0129200), while increased transcripts accumulated in the control samples in the other. One (OS05G0375400) of the 6 PR2 genes was induced in the control samples, and 5 genes (OS01G0940700, OS01G0940700, OS07G0539900, OS07G0539900, OS07G0539100) were induced in the treated samples. The only PR3 gene (OS10G0542900) detected was up-regulated in the treated samples and down-regulated in the control samples. As for PR5 and PR10, all genes 


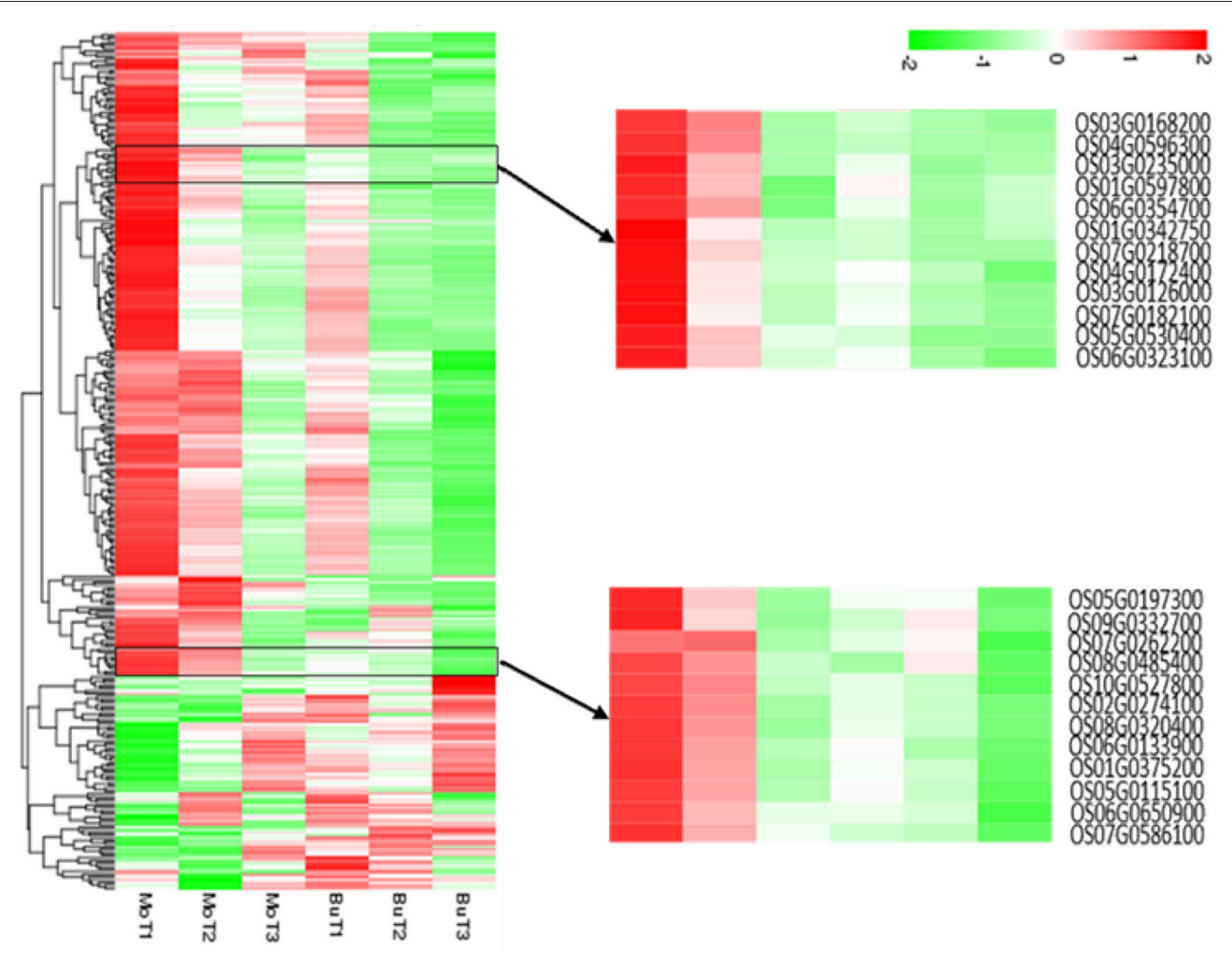

FIGURE 2 | Hierarchical clustering of the differentially expressed genes. The heat map shows the gene expression clusters and sample clusters. Each line indicates data from a single gene. The color bar represents the $\log _{10}(\mathrm{RPKM})$ of each gene, ranging from green ( -2$)$ to red (2). Two detailed gene clusters show the genes that are up-regulated in the MoHrip1-treated samples after 24 and $48 \mathrm{~h}$ and down-regulated in the buffer-treated samples after 24,48 , and $72 \mathrm{~h}$.

detected (OS12G0628600, OS12G0569500, OS12G0555000, OS12G0555200) were induced both in the treated and control samples, while the level of transcript accumulation in the control samples was much lower (Figure 3A).

\section{WRKY, NAC, and the ERF Transcription Factor}

Transcriptional control of the expression of resistance genes plays a crucial role in the response to stress in plants. Transcription factors have been identified as important for regulating defensive gene expression (Singh et al., 2002). In our research, WRKY, NAC, AP2/ERF were three types of transcription factors (TFs) detected, all of which were found to participate in the regulation of plant defense genes (Gutterson and Reuber, 2004; Wei et al., 2013; Sun et al., 2015). Two genes (OS06G0649000, OS01G0584900) encoding WRKY TFs were detected with increased transcript accumulation in the treated samples, while no changes were observed in the control samples. The remaining 6 genes (OS01G0246700, OS01G0246700, OS05G0322900, OS05G0343400, OS05G0474800, OS02G0181300) encoding WRKY TFs were detected in both groups but were up-regulated only in the treated groups.

The two NAC-related genes (OS01G0816100, OS01G0816100) detected were induced in the treated rice.
All four genes encoding the ERF TFs were up-regulated after treatment, except for one (OS04G0546800), which was mainly down-regulated after treatment (Figure 3B).

\section{Antifungal-Related Proteins}

Phytoalexins, ATP-binding cassette (ABC) transporters, and peroxidases are three types of compounds whose encoding genes were detected in our data, and all are known to be involved in reducing pathogen-related damage. Then, we classified these compounds as antifungal-related proteins. Phytoalexins play important roles in the disease resistance of various plant species, and diterpenes comprise a major portion of the phytoalexins, which are induced in rice after infection with pathogenic fungi or a pathogen-derived elicitor (Duan et al., 2014; Umemura et al., 2014). All the phytoalexin synthesis-related genes were detected in at least one treated and control group and accumulated to higher transcript levels in rice under the treatment but not the control conditions, except for one gene (OS03G0225900), which was induced in the treated group and repressed in the control group (Figure $3 \mathrm{C}$ ).

Previous studies have shown that $\mathrm{ABC}$ transporters are associated with various host-pathogen interactions. The pleiotropic drug resistance (PDR) subfamily of plant ABC transporters has been implicated in plant defense (Jasiński 


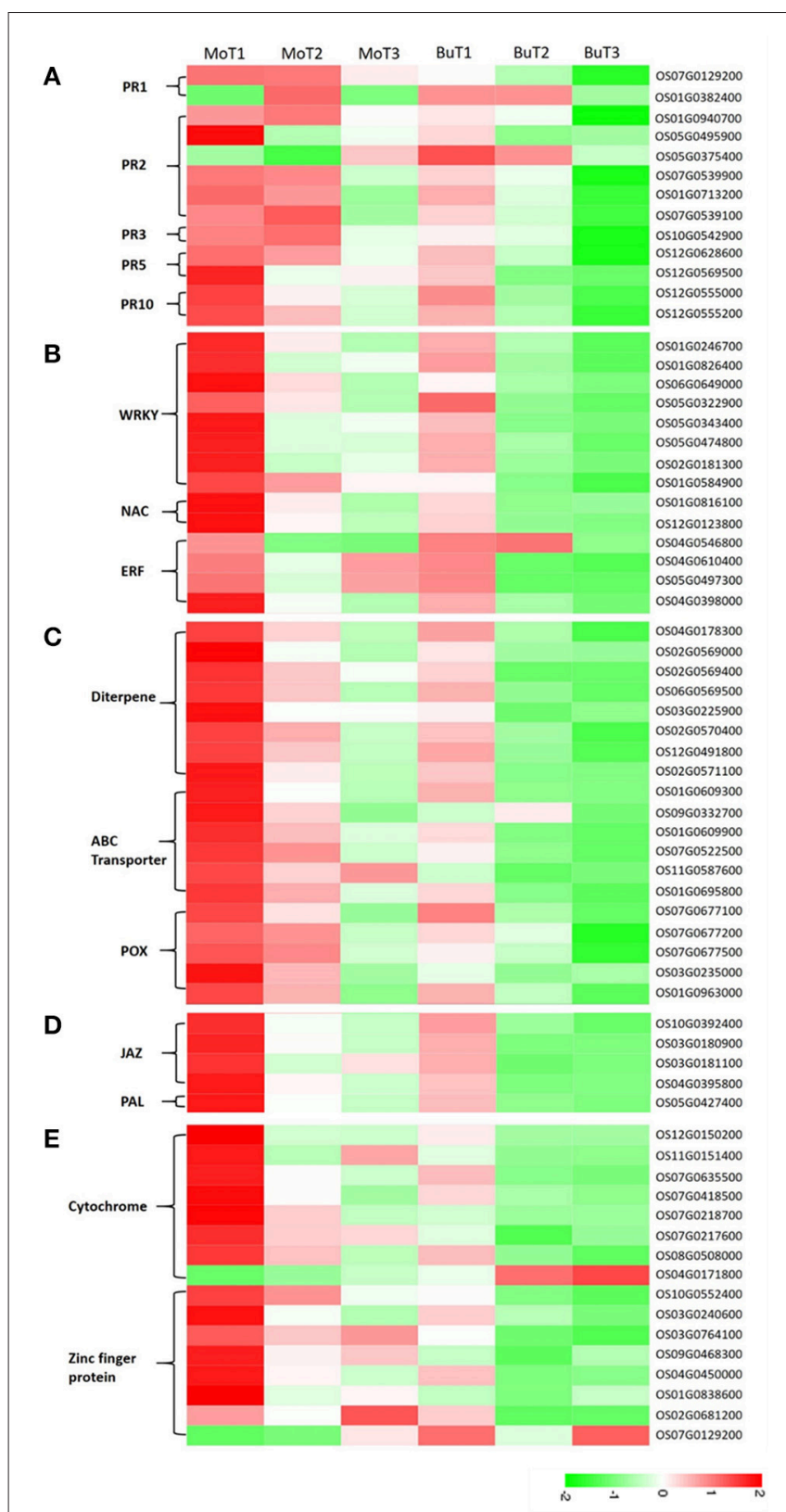

FIGURE 3 | Heat maps show increased or decreased gene transcripts in rice following MoHrip1 and buffer treatment, using the corresponding non-treated samples as controls. The bottom color bar represents the $\log _{10}$ of the RPKM values for each gene, ranging from green $(-2)$ to red (2). A comparison of the gene expression levels in rice after treatment with MoHrip1 and buffer for the PR proteins (A); transcription factors (B); phytoalexin-, ABC transporter-, and peroxidase-related genes (C); SA and JA pathways (D); and CYP450- and ZFP-related genes (E)

et al., 2001; Campbell et al., 2003). In the 6 genes encoding $\mathrm{ABC}$ transporters (Figure 3C), half were induced and half were repressed in the rice under control conditions. However, all of the genes were up-regulated and obtained a higher level of expression in the rice after treatment than in the control rice samples.
Increased peroxidase (POX) activity has been observed in a number of resistant interactions involving plant-pathogen interactions. A previous study demonstrated that POXs were upregulated in rice when infected by $M$. oryzae (Chittoor et al., 1997; Rauyaree et al., 2001). Five genes related to POX were detected in our data (Figure 3C). Two genes (OS07G0677500, OS03G0235000) were repressed in the control but induced in the treatment samples. The expression levels of the other 3 genes (OS07G0677100, OS07G0677200, OS01G0963000) in the groups showed that both the treatment and control conditions could induce gene expression, but that MoHrip1 produced a more intense effect in up-regulating the genes. These findings suggest that phytoalexin, the $\mathrm{ABC}$ transporter and POX might be involved in the resistance induced by MoHrip1 in rice.

\section{Salicylic Acid and Jasmonic Acid Pathways}

SA and jasmonic acid (JA) are two major phytohormones responsible for regulating plant defenses against various pathogens (Bari and Jones, 2009; Thaler et al., 2012). SA biosynthesis derives from chorismate, an intermediate of plant phenylpropanoid pathway, then the phenylalanine ammonia lyase (PAL)-mediated pathway. PAL is a key regulator of the phenylpropanoid pathway and also plays an important role in regulating SA biosynthesis. The core events of JA pathway are defined after hormone perception by $\mathrm{SCF}^{\mathrm{COI}}$, JAZ (JAsmonate ZIM domain) repressors are targeted for proteasome degradation, releasing MYC2 and de-repressing transcriptional activation (Chaman et al., 2003; Chico et al., 2008). All genes encoding JAZs (OS10G0392400, OS03G0180900, OS03G0181100, OS04G0395800) had relatively low expression levels in rice under control conditions and relatively high expression levels in rice after MoHrip1 treatment. The PAL-encoding gene (OS05G0427400) has the same expression patterns in rice under the treatment and control conditions with the JAZs (Figure 3D). Therefore, it can be implied that SA pathway was activated and the JA pathway was blocked in rice treated with MoHrip1.

\section{Cytochrome and Zinc-Finger Protein}

The cytochrome P450 (CYP450) family is a superfamily of enzymes that participate in metabolism and form antibiotic compounds (Zhou et al., 1999; Nomura et al., 2002). In our data, eight genes related to CYP450 were detected. Four genes accumulated higher levels of transcripts in the treated rice, and these genes were induced in both the treated and control groups. Three genes were induced in the rice under treatment and were repressed in the control rice. One gene (OS04G0171800) was induced under control conditions and repressed under treatment conditions (Figure 3E).

Zinc-finger proteins (ZFPs) form a transcription factor family known to be involved in various forms of abiotic stresses, such as drought, high salinity, hot, and cold, according to many studies. However, the available research on their responses to biotic stresses remains limited (Huang et al., 2007; Islam et al., 2009). Four genes encoding ZFPs were repressed in rice under control conditions and were induced in rice under treatment conditions. The other 4 genes encoding ZFPs were 
induced in rice under control conditions but accumulated more transcripts in rice under treatment conditions, except for one gene (OS07G0129200), which had lower transcript levels in the treated rice (Figure 3E). The responses of CYP450 and ZFP to the elicitors indicated that these genes might be involved in contributing to the induced resistance and could be considered candidate resistance genes.

\section{The Identification of Genes Induced Specifically by MoHrip1 in Rice}

In our data, we have identified 80 genes with particular expression patterns in the MoHrip1-treated group and no similar expression patterns in the buffer-treated group. These genes can be classified into five groups (Figure 4): Figure 4A, significantly induced in the MoHrip1-treated rice at 24 hat with low or undetected expression in the other conditions; Figure 4B, significantly induced in the MoHrip1-treated rice at 48 hat with low or undetected expression in the other conditions; Figure 4C, significantly induced in the MoHrip1-treated rice at 72 hat with low or undetected expression in the other conditions; Figure 4D, significantly induced in the MoHrip1-treated rice at 24 and 48 hat with low or undetected expression in the other conditions; Figure 4E, significantly induced in the MoHrip1-treated rice at 48 and 72 hat with low or undetected expression in the other conditions. These results may provide data for discovering genes that can interact with MoHrip1 in rice.

Nine genes (indicated by arrows in Figure 4) that were up-regulated in MoHrip1-treated rice and not expressed in the buffer-treated rice were selected for qRT-PCR validation (Figure 5). These genes include three leucine-rich repeat proteins, an EF-hand type protein, a salicylic acid-binding protein, an ABC-transporter-like protein, two ZFPs and one heat shock protein (Table S5). The qRT-PCR results were consistent with the DGE data, as all gene expression levels in the rice under the control conditions were extremely low compared to those in the rice under treatment at certain time intervals. The 9 genes were verified as induced by MoHrip1 in rice and may be related to the resistance of rice induced by the elicitor. These genes can be selected as MoHrip1 interaction genes for further study.

\section{Endogenous Levels of SA in Rice}

SA plays an important role in the plant immune response, and significant progress has been made in recent years in understanding the SA-mediated defense response (An and Mou, 2011). Previous research has led to a popular belief that SA and JA have antagonistic interactions (De Vleesschauwer et al., 2013). In this experiment, the SA levels in rice under the treatment and control conditions were measured as the MoHrip1-treated rice showed enhanced resistance to blast according to the results of a previous study (Figure 6). Moreover, the sequencing data indicate that the JAZ genes were induced significantly in rice under treatment conditions. JAZs were the repressors of the JA signaling and were involved in a negative regulatory feedback loop of the JA transduction pathway (Chico et al., 2008).

The SA levels detected in rice under the control conditions were not very different, while the SA levels in rice under the treatment conditions were altered significantly during the time intervals we assessed. The SA levels in MoHrip1-treated rice

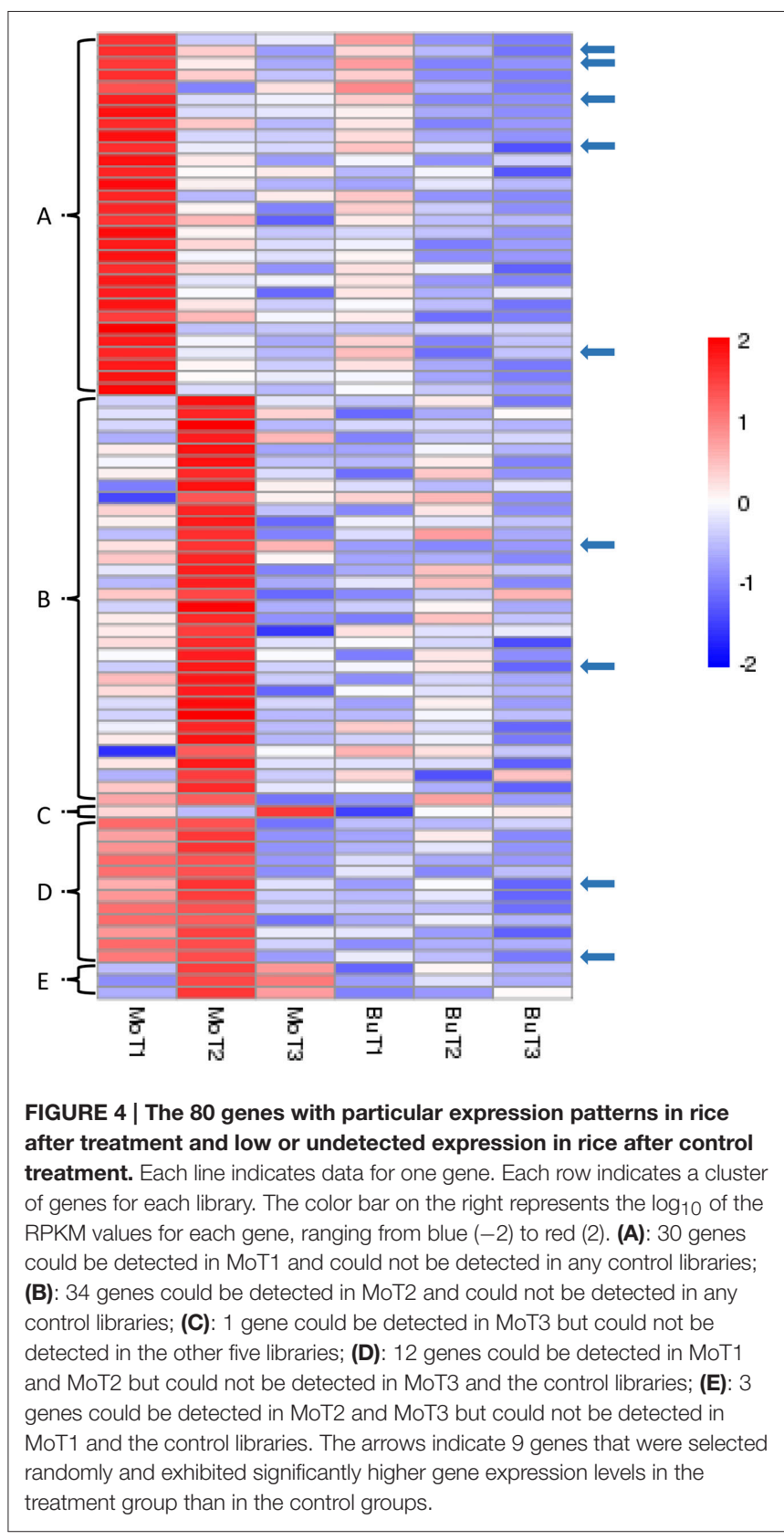

decreased slightly at 12 hat and increased prominently to their highest level at 24 hat. The SA levels started to decrease at 36 and 48 hat, began to increase at 60 hat and finally decreased again at 72 hat. Intriguingly, the SA levels of the rice under the treatment conditions across all time intervals were higher than those of the rice under control conditions, excluding 48 hat, at which the SA levels reached their lowest point. This result can be explained through the feedback regulation of SA, which was likely induced significantly by MoHrip1.

\section{Endogenous Levels of GA in Rice and Growth Promotion}

Gibberellins (GAs) are a type of essential phytohormone and control many aspects of plant growth and development. Recent 


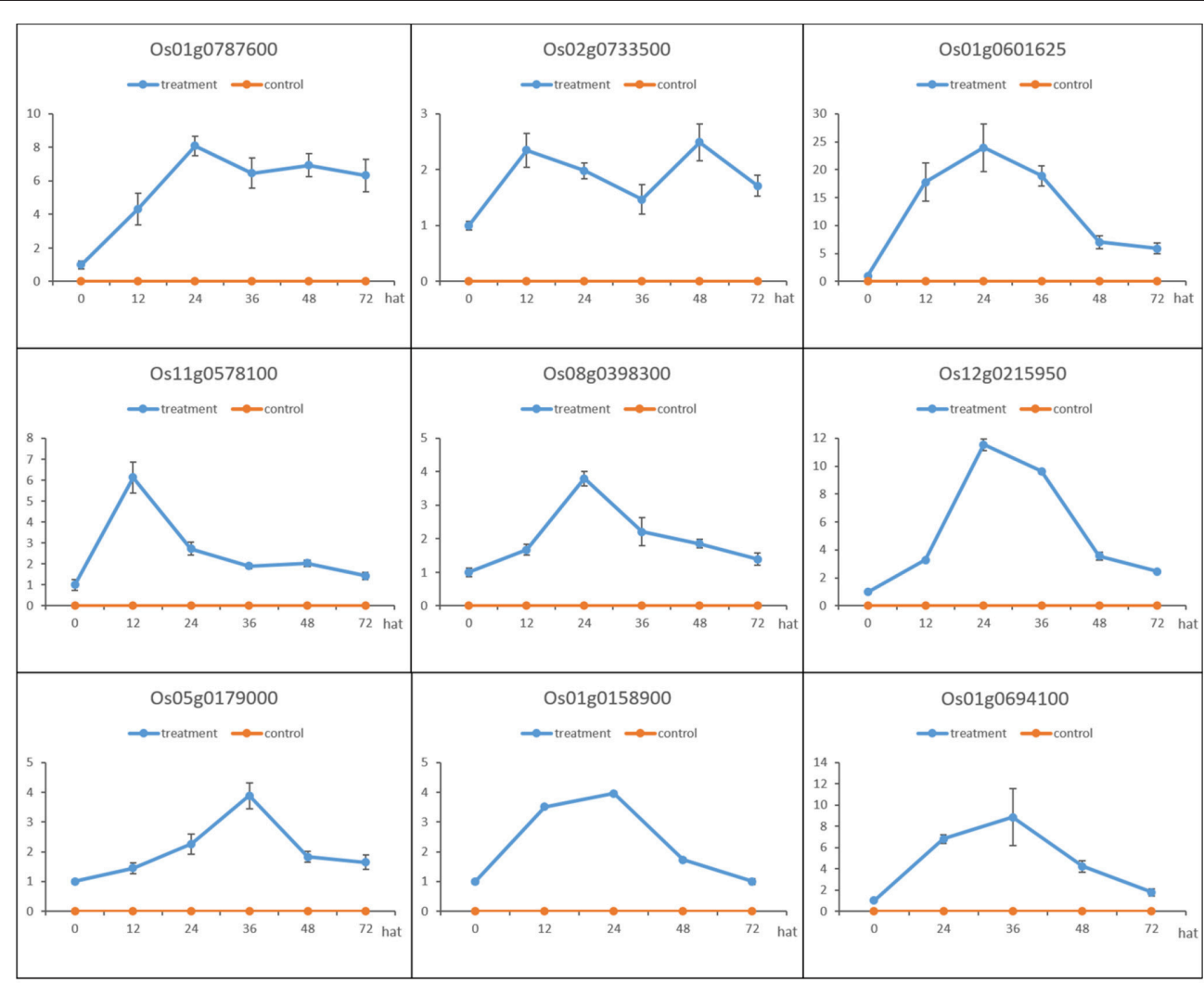

FIGURE 5 | Validation of the DGE results by qRT-PCR of the 9 genes that were up-regulated in the MoHrip1-treated rice but were not expressed in the buffer-treated rice. The expression levels on the $y$-axis were relative to the non-treated rice (NoTO) after normalization with the $\beta$-actin gene. This experiment was repeated three times, and data are presented as the average $\pm S D$ with $n=3$; hat: $h$ after treatment.

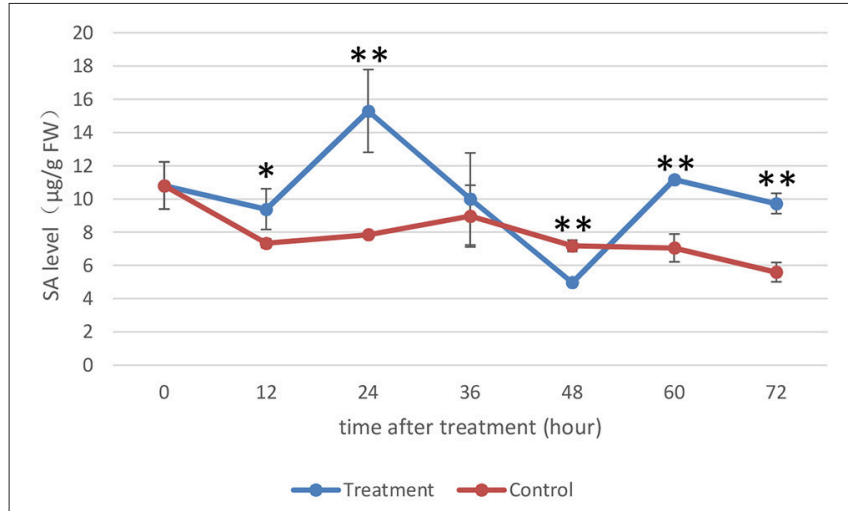

FIGURE 6 | Endogenous SA levels of rice. The SA levels ( $\mu \mathrm{g} / \mathrm{g}$ FW) on the $y$-axis were relative to those of non-treated rice (NoTO). The experiment was repeated three times, and the data are presented as the average $\pm S D$ with $n$ $=3$. Asterisks indicate statistically significant differences, as measured using SAS software (Duncan's $t$ test, ${ }^{* *} p<0.01,{ }^{\star} p<0.05$ ).

studies have revealed that crosstalk between GA and JA signaling is involved in both plant development and defense. Moreover, the crosstalk is regulated by interaction between the DELLA and JAZ proteins as repressors to modulate the activity of relative transcriptional factors in response to the GA and JA signals (Hou et al., 2013). We predicted that the induced JAZs might indirectly affect GA levels. The endogenous GA levels were then measured, along with the length and weight of the rice under the treatment and control conditions (Figures 7, 8). All measured data indicated that 5 $\mu \mathrm{g} / \mathrm{ml}$ was the best concentration of MoHrip1 to produce the greatest effect on promoting the growth of rice. According to this experiment, the morphological differences observed at each concentration of MoHrip1 started 9 days after treatment (Figures 7A-C, 8). Moreover, the phytohormone GA also required 9 days for growth promotion to be observed at a significant level (Figure 7D). These results indicate that MoHrip1 may influence the level of GA and that there is an optimum concentration at which GA is most effective in regulating plant growth.

\section{Induction of Resistance to Rice Blast by MoHrip1}

Three groups of rice plants were sprayed with $M$. oryzae spore suspension (strain KJ201) after 3 days of incubation with either MoHrip1 or Tris-Cl. Screening was carried out on detached leaves. The lesions on the leaves of CK group exhibited typical and severe symptoms at 7 days after inoculation. At this time, 


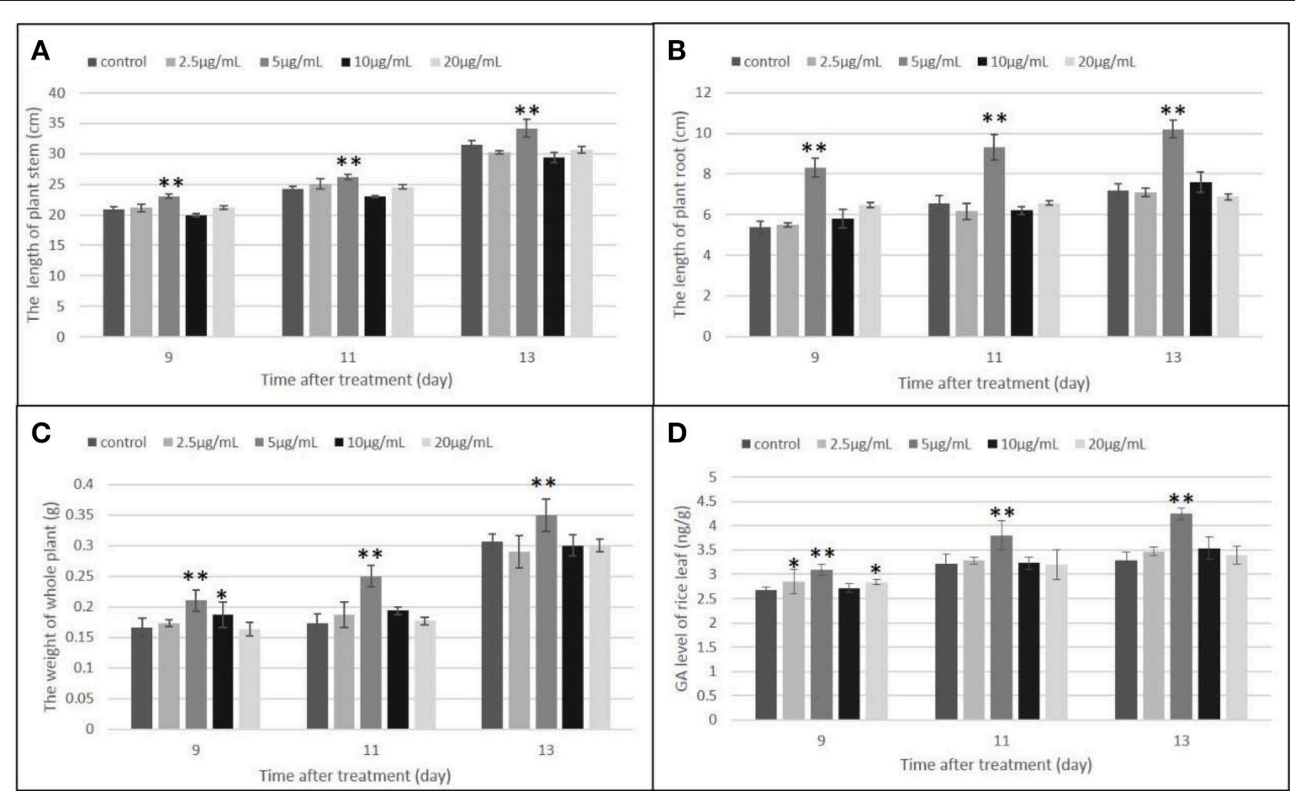

FIGURE 7 | Growth promotion and GA level of plant seeds treated with a range of concentrations of MoHrip1. (A) Average length of the plant stem. (B) Average length of the plant root. (C) Average weight of the whole plant. (D) GA levels of plants under different treatment conditions. Values are presented as the mean $\pm S D$ of three replicates, each consisting of 15 seedlings. Asterisks indicate statistically significant differences, as measured using SAS software (Duncan's $t$ test, ${ }^{* *} p$ $\left.<0.01,{ }^{*} p<0.05\right)$.
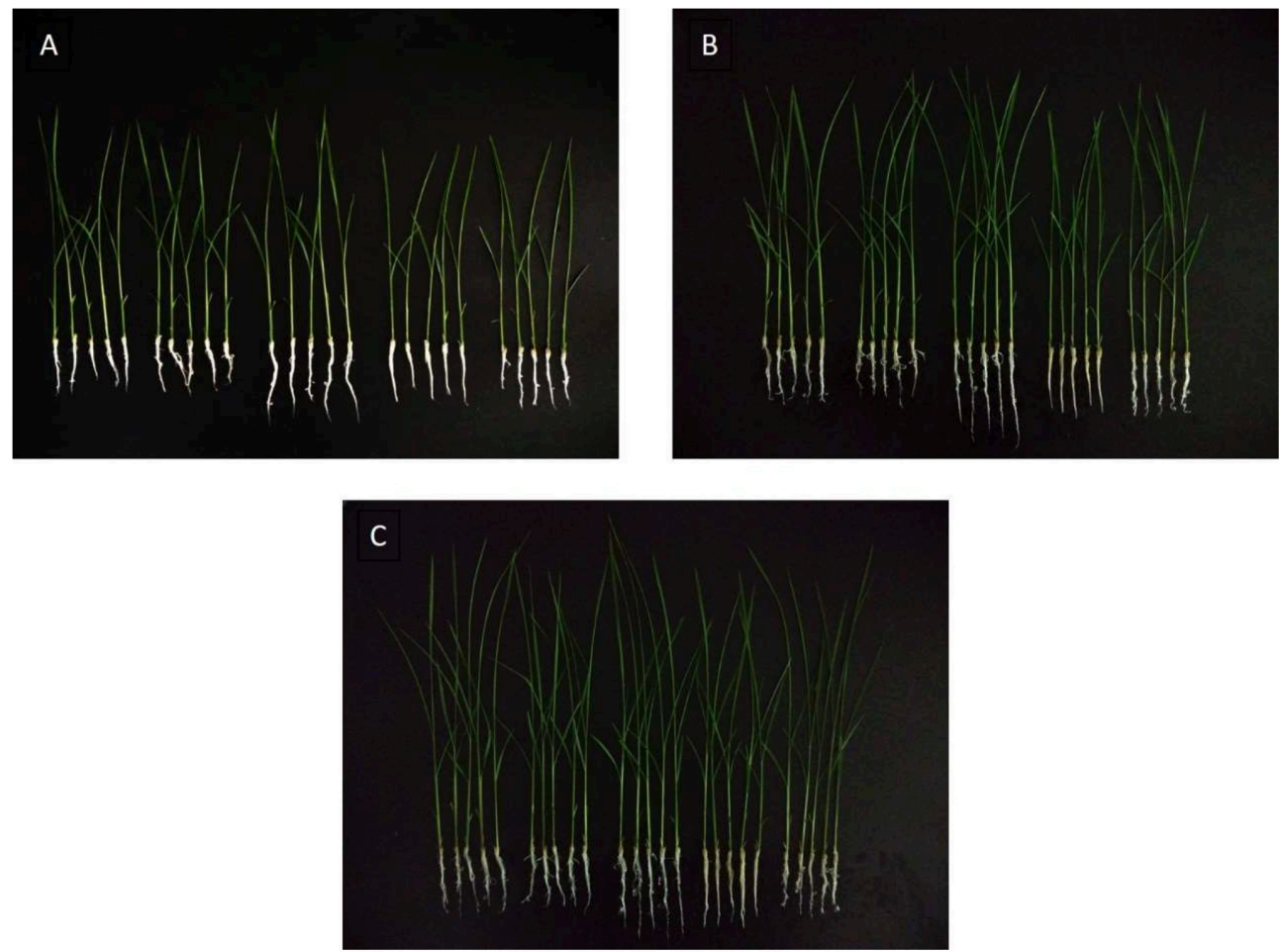

FIGURE 8 | Rice seeds treated with a range of concentrations of MoHrip1 show significant differences in their growth morphological characterization. (A) Rice seed germination after 9 days of treatment. (B) Rice seeds germinated after 11 days of treatment. (C) Rice seeds germinated after 13 days of treatment. The group arranged horizontally in each graph indicates the different concentrations of MoHrip1 used to treat the rice seeds $(0,2.5,5,10$, and 20 $\mu \mathrm{g} / \mathrm{mL}$, from left to right). 


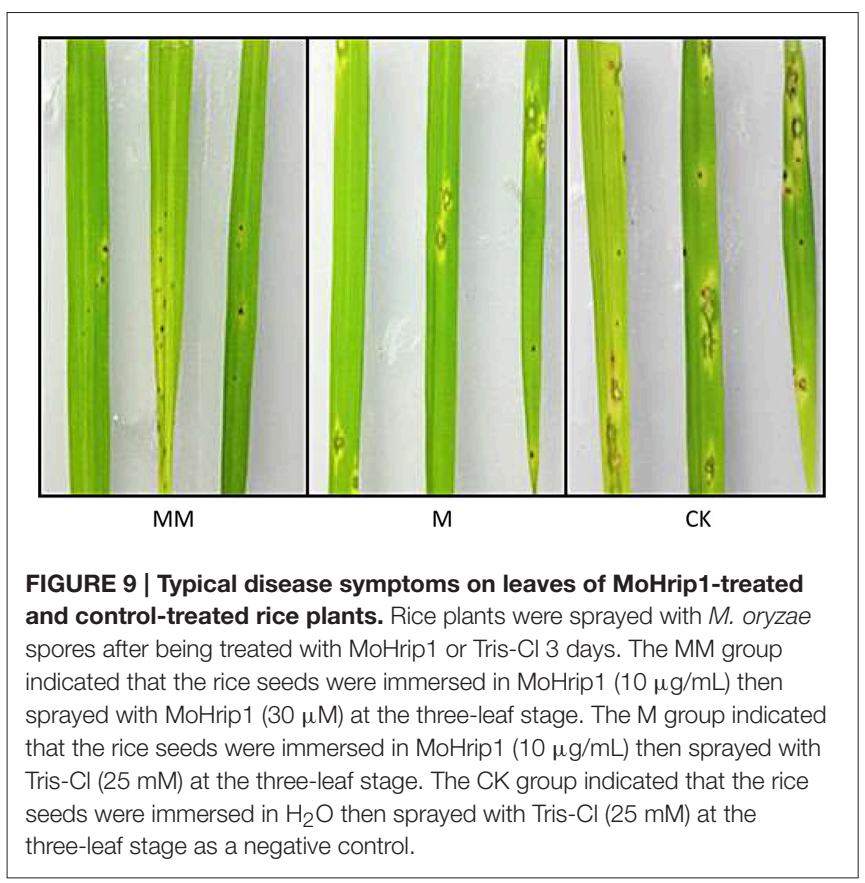

TABLE 3 | Disease severity of rice blast in leaves of MoHrip1-treated and control-treated rice plants.

\begin{tabular}{|c|c|}
\hline Rice seedling samples & Rice blast score \\
\hline $\begin{array}{l}\text { MM (Rice immersed in MoHrip1 and sprayed } \\
\text { with MoHrip1) }\end{array}$ & $3.126 \pm 0.212 \mathrm{Aa}$ \\
\hline $\begin{array}{l}\text { M (Rice immersed in MoHrip1 and sprayed } \\
\text { with Tris-Cl) }\end{array}$ & $3.734 \pm 0.173 \mathrm{Ab}$ \\
\hline $\begin{array}{l}\mathrm{CK} \text { (Rice immersed in } \mathrm{H}_{2} \mathrm{O} \text { and sprayed with } \\
\text { Tris-Cl) }\end{array}$ & $5.862 \pm 0.285 B$ \\
\hline \multicolumn{2}{|c|}{$\begin{array}{l}\text { The disease scores of the rice seedlings were evaluated on a scale of } 0-9 \text { at } 7 \text { days post- } \\
\text { inoculation. Values are presented as the mean } \pm S D \text { of three replicates, each consisting } \\
\text { of } 25 \text { seedlings. Values with different letters indicate statistically significant differences } \\
\text { (Duncan's } t \text { test, different capital letters means } p<0.01 \text {, different small letters means } \\
p<0.05 \text { ). }\end{array}$} \\
\hline
\end{tabular}

only small and constrained lesions were observed on the leaves of both MM group and M group, and most of the plants remained green and healthy. While the constrained lesions on leaves of MM group were smaller than those on leaves of $\mathrm{M}$ group (Figure 9). The disease severity of all plants from the three groups was evaluated on a standard international $0-9$ scale at 7 days post-inoculation, the leaf blast score of MM was a little lower than that of M group. While the blast score of MM group and $\mathrm{M}$ group was much lower than that of CK group (Table 3). Furthermore, we measured the root length of rice plants after we examined the disease indices. MM group and $\mathrm{M}$ group exhibited obvious function of MoHrip1 in growth promotion (Figure S8). Taken together, the results from the disease-resistance assay and growth promotion assay reconfirmed the inference that MoHrip1 could not only increase rice plant resistance to the rice blast but also promote plant growth.

\section{DISCUSSION}

\section{Advantages and Defects of DGE in the Gene Expression Profiling Study}

In the early stage of studies, elicitor-responsive genes were identified by means of differential mRNA display analysis, DNA microarrays, and other methods (Kim et al., 2000; Day et al., 2002). These methods were later replaced by NGS-based transcriptome profiling, which is based on the sequencing of relatively short reads, with extensive sequence data to allow for the detection and quantification of rare or novel gene discovery (Morrissy et al., 2009). DGE, as used in this study, includes profiles based on NGS, generates digital rather than simulated gene expression measurements and avoids many of the inherent limitations of other analysis methods (Xiao et al., 2013).

DGE has already been used for studying genes in many species, such as wheat, sweet potato, and rice (Tao et al., 2012; Xiao et al., 2013). In general, current studies aiming at resistance-related genes in rice, which introduced NGS-based technology, use two styles of research model: a pathogen vs. whole plant model or a pathogen secretion vs. rice suspension cell model (Day et al., 2002; Bagnaresi et al., 2012). In this study, MoHrip1, a protein elicitor secreted by M. oryzae, and the whole rice plant form a new style of research model. We obtained sufficient data from the Illumina sequencing experiment (Table 1) to allow for gene detection and applied realtime PCR to validate the DGE veracity (Figure 1). A total of 308 genes were differentially expressed, and 80 genes were expressed in the treatment condition but not in the control condition. These findings indicate that pathogen secretion (elicitor) vs. the whole plant could constitute a third research model and that DGE could fulfill the technical requirements needed to identify potentially interesting genes. This technology also has its drawbacks. DGE was a method designed to identify DEGs based on relatively short sequence reads. Therefore, the differences between the experimental and control groups are necessary; genes with similar expression levels in the groups but acted functionally might be ignored. In conclusion, our results provide an overview of the gene expression patterns of elicitor-treated rice and offer a valuable set of data for the further discovery of candidate genes that interact with the elicitor.

\section{SA Played a Major Role in the Blast-Resistance of Rice and May Be Regulated by MoHrip1}

M. oryzae is a hemibiotrophic pathogen, as its invasion begins with a biotrophic phase followed by necrotrophic phase, leading to host cell death (Talbot, 2003). In general, the JA pathway is associated with defense against necrotrophic pathogens, while the SA pathway is thought to be involved in the plant resistance to biotrophic and hemibiotrophic pathogens (Bari and Jones, 2009). The positive role of the SA pathway in blast-resistance has been reported (Iwai et al., 2007; Daw et al., 2008). The SA pathway includes two steps: SA biosynthesis followed by SA signal transduction, which leads to the activation and expression of SA-responsive genes (Ellis and Amrhein, 1971; Zhu-Salzman 


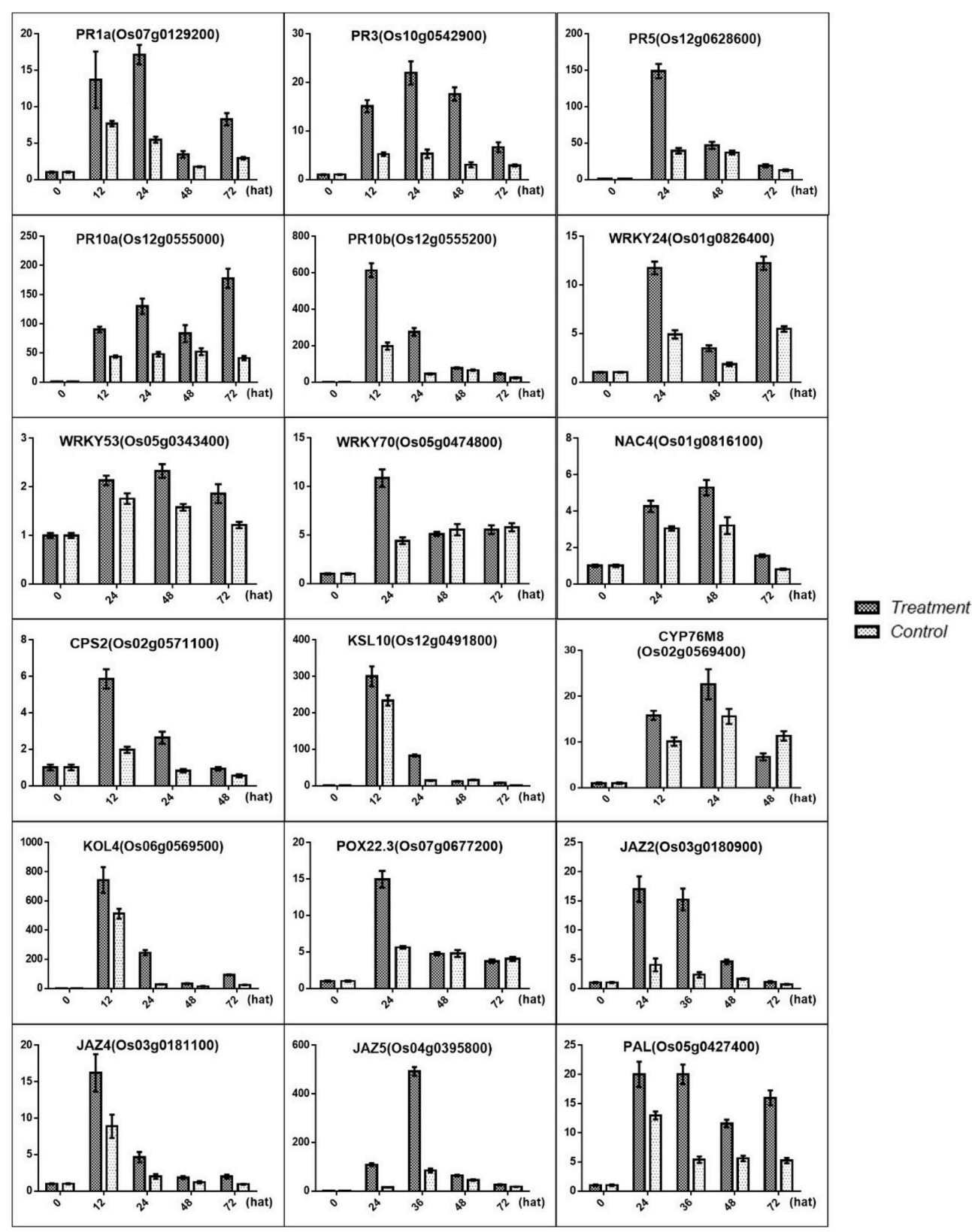

FIGURE 10 | Validation of the DGE transcriptome results by qRT-PCR for the genes involved in the resistance induced by MoHrip1 in rice. The expression levels on the $y$-axis were relative to those of the non-treated samples $(0 \mathrm{~h})$ after normalization with the rice $\beta$-actin gene. The experiment was repeated three times, and the resulting data are presented as the average $\pm S D$, with $n=3$; hat: h after treatment.

et al., 1998). The JA pathway possesses two similar steps to the SA pathway (Fonseca et al., 2009).

Based on our data, the SA biosynthesis pathway was activated, and the result was confirmed by qRT-PCR for PAL (OS05G0427400), which was involved in SA biosynthesis. In contrast, the JA signal transduction pathway was blocked, and this result was confirmed by qRT-PCR for the JAZ2 (OS03G0180900), JAZ4 (OS03G0181100), and JAZ5 (OS04G0395800) repressors of JA signal transduction (Figure 10). One SA biosynthesis-associated gene and 3 JA pathway repressor genes were significant differentially expressed.
As the antagonistic function hypothesis for the SA-JA crosstalk research prevailed, and the endogenous SA levels in the treated condition were obviously higher than those in the control condition, it can be predicted that SA biosynthesis was induced in rice treated with MoHrip1.

Previous studies have identified some PR genes involved in pathogen resistance located downstream of the SA pathway that are regulated by the SA signals (Agrawal et al., 2001; McGee et al., 2001; Rakwal et al., 2001). These PR genes were also found to be up-regulated, and this result was confirmed by qRT-PCR for PR1a (OS07G0129200), PR3 (OS10G0542900), PR5 
(OS12G0628600), PR10a (OS12G0555000), and PR10b/PBZ1 (OS12G0555200) (Figure 10). Furthermore, transcription factors were another important part of regulators involved in pathogen resistance. Some TFs were found to act as a node between the SA and JA pathway, which activates SA-induced genes and represses JA-induced genes ( $\mathrm{Li}$ et al., 2004; Shimono et al., 2007). Some TFs are involved in the plant defense response along with SA (Kaneda et al., 2009). Some TFs associated with SA were induced in rice under treatment conditions, and the results were verified by qRT-PCR for WRKY24 (OS01G0826400), WRKY53 (OS05G0343400), WRKY70 (OS05G0474800), and NAC4 (OS01G0816100) (Figure 10).

An increasing number of studies has shown that rice possesses a high basal level of SA. Although the primary function of SA may not be to induce the expression of defense genes, it still appears to protect rice from damage during pathogen infection (Yu et al., 1997; Yan and Dong, 2014). From our results, even though the SA content increased, no significant difference was noted in the rice in the treatment group compared with the rice in the control group. Furthermore, a large amount of resistance-related substances, which were associated with SA, were obviously accumulated. It can be assumed that an increased level of SA may not only provide an effective signal to induce gene expression but also play an important role in reducing pathogeninduced damage. Moreover, our results verified the importance of the SA pathway in blast-resistance.

\section{Phytoalexin May Be Important in Blast-Resistance Induced by MoHrip1}

Phytoalexins include a range of secondary metabolites produced in rice upon $M$. oryzae infection that function in the defense system (Wang et al., 2012; Miyamoto et al., 2014). The rice phytoalexins have been identified to fall into two groups, and the diterpenoid-type phytoalexins formed the major portion of these substances. All phytoalexin-related genes that were up-regulated in our data were involved in the biosynthesis of phytocassanes A-E and oryzalexin A-F. Oryzalexin is considered a biologically important phytoalexin, as it has high antimicrobial activity in in vitro assays. There is no direct evidence to prove its function in blast-resistance. The two types of phytoalexin shared the same precursor. Our results showed that phytoalexin biosynthesis related genes were increased in rice after treatment with MoHrip1, and the results were confirmed by qRT-PCR for CPS2 (OS02G0571100), KSL10 (OS12G0491800), KOL4 (OS06G0569500), and CYP76M8 (OS02G0569400). These findings suggest that phytoalexins, especially Oryzalexin, may be important in the blast-resistance induced by MoHrip1 and that phytocassanes may be involved in the defense response.

\section{GA May Be Induced to Promote Plant Growth through the Crosstalk between Della and Jaz, Which Is Induced By MoHrip1}

GAs are plant hormones that play pivotal roles in promoting growth. Current research suggests that GA promotes plant growth by regulating the degradation of DELLAs, a class of nuclear growth repressors. High levels of DELLAs will provide feedback that negatively controls GA levels, while low levels of DELLAs may stimulate GA production to obtain a balance between the GAs and DELLAs (Claeys et al., 2014). Previous studies have demonstrated that the GA-signaling repressor DELLA and the JA-signaling repressor JAZ engage in crosstalk that modulates the action between GA and JA to regulate plant growth (Yang et al., 2012). According to our data, the JAZs are induced by MoHrip1, followed by more extensive crosstalk between the JAZs and DELLAs. Although the level of DELLAs was not measured, it can be predicted that increased JAZs led to DELLA repression based on the GA contents measured in this experiment, resulting in an overall growth promotion in rice. The results were confirmed by qRT-PCR (Figure 10) and morphologic testing (Figure 7).

Intriguingly, the GA and phytoalexin synthesis pathways shared the same precursors: geranylgeranyldiphosphate (GGDP) and ent-copalyldiphosphate (ent-CPP). However, there appears to be separate sets of ent-CPP synthases for gibberellin (KS1) vs. phytoalexin (KSL7, KSL10) biosynthesis. There was no evidence to demonstrate that KS1 was induced in the rice after treatment, while the genes encoding KSL7 and KSL10 were confirmed to be up-regulated under treatment. These findings suggested that the increased GA levels did not result from the sequential catalysis derived from GGDP and were instead affected by the crosstalk between the GA and JA pathway repressors caused by MoHrip1.

\section{CONCLUSION}

We present an RNA-seq study focusing on the response of rice to secretion protein elicitors. Our transcriptome data provide comprehensive insight into the gene expression profiles induced by MoHrip1 in rice. SA was the main phytohormone induced to participate in blast-resistance, and the JA pathway was repressed in rice after treatment with MoHrip1. Phytoalexins were induced to synthesized and played an important role in reducing the damage caused by pathogen infection. The GA content increased to promote plant growth after rice was treated with MoHrip1. There was a balance and exchange between the plant defense response and plant growth promotion. The DGE data revealed that the protein elicitor MoHrip1 possessed the potential not only to protect plants by inducing pathogenresistance but also to regulate plant growth indirectly, even though involvement with some other aspects of the plant life cycle. Nine genes were identified as candidates, and it would be of great value to further characterize their roles in interactions with MoHrip1.

\section{AUTHOR CONTRIBUTIONS}

SL, ZW, and HZ designed research; SL and ZW performed research; SL analyzed data and wrote the paper; XY, LG, DQ, and $\mathrm{HZ}$ revised this paper. 


\section{FUNDING}

This work was supported by grants from the National Hi-Tech Research and Development Program of China ("863" Projects, Grant Nos. 2012AA101504).

\section{SUPPLEMENTARY MATERIAL}

The Supplementary Material for this article can be found online at: http://journal.frontiersin.org/article/10.3389/fpls.2016. 01818/full\#supplementary-material

Figure S1 | Saturation curve of DGE sequencing. Quantified gene expression with $10,20,30 \ldots . .90 \%$ of the total mapped reads as the final scores. Comparing the RPKM of one gene in each score with the final gene expression level means that genes can be quantified accurately in this score when the deviation is less than $15 \%$. The $X$-axis represents the percentage of reads mapping to the genome, while the $Y$-axis represents the ratio of genes with a qualify error of $15 \%$. The pictures from (A-N) represent NoT0_1, NoT0_2, MoT1_1, MoT1_2, BuT1_1, BuT1_2, MoT2_1, MoT2_2, BuT2_1, BuT2_2, MoT3_1, MoT3_2, BuT3_1, and BuT3_2, respectively.

Figure S2 | Pearson correlation coefficients between samples. The axis represents the gene expression levels of one sample in two replicates under the same condition. (A-G) represent the correlations between the two replicates of the NoT0, BuT1, MoT1, BuT2, MoT2, BuT3, and MoT3 groups, respectively.

Figure S3 | Histogram of GO enrichment. The x-axis indicates the number of genes enriched in terms, while the $y$-axis indicates the enriched GO terms. Different colors represent biological process (green), cell component (blue) and molecular function (red). *Indicates GO terms with significant enrichment. (A-C) Represent the GO enrichments of the differentially expressed genes in rice after 1 , 2 , and 3 days of treatment, respectively.

Figure S4 | Scatter diagram of the KEGG enrichment. The $y$-axis indicates the name of the pathway, and the x-axis represents the Rich factor. The spot size indicates the number of differentially expressed genes in the pathway. The $q$-value represents the corrected $p$-value, and a $q<0.05$ indicates significant enrichment. (A-C) Represent the pathway enrichment of differentially expressed genes in rice after 1,2 , and 3 days of treatment, respectively.

Figure S5 | Volcano plot. The volcano plot indicates the expressed genes, and red or green spots indicate genes with significant expression differences. Red indicates up-regulated genes, while green indicates down-regulated genes. Blue spots indicate genes without significant expression differences. The $\mathrm{x}$-axis

\section{REFERENCES}

Agrawal, G. K., Rakwal, R., Jwa, N. S., and Agrawal, V. P. (2001). Signalling molecules and blast pathogen attack activates rice OsPR1a and OsPR1b genes: a model illustrating components participating during defence/stress response. Plant Physiol. Biochem. 39, 1095-1103. doi: 10.1016/S0981-9428(01)01333-X

An, C., and Mou, Z. (2011). Salicylic acid and its function in plant immunityF. J. Integrat. Plant Biol. 53, 412-428. doi: 10.1111/j.1744-7909.2011.01043.x

Anders, S. (2010). HTSeq: Analysing High-throughput Sequencing Data with Python. Available online at: http://www.huber.embl.de/users/anders/HTSeq/ doc/overview.html

Anders, S., and Huber, W. (2010). Differential expression analysis for sequence count data. Genome Biol. 11:R106. doi: 10.1186/gb-2010-11-10-r106

Atzorn, R., and Weiler, E. W. (1983). The immunoassay of gibberellins: II. Quantitation of GA3, GA 4 and GA 7 by ultra-sensitive solid-phase enzyme immunoassays. Planta 159, 7-11. doi: 10.1007/BF00998807

Bagnaresi, P., Biselli, C., Orrù, L., Urso, S., Crispino, L., Abbruscato, P., et al. (2012). Comparative transcriptome profiling of the early response to Magnaporthe oryzae in durable resistant vs. susceptible rice (Oryza sativa L.) genotypes. PLoS ONE 7:e51609. doi: 10.1371/journal.pone.0051609 indicates the fold change in the gene expression levels in the different samples. The $y$-axis indicates the statistical significance of the gene expression differences. MoT1 vs. BuT1, MoT2 vs. BuT2, and MoT3 vs. BuT3 represent the comparison between the treatment and control rice groups after MoHrip1 treatment for 1, 2, and 3 days.

Figure S6 | Venn diagrams of the groups. (A) Differentially expressed genes among the three groups of treated and control samples superimposed. (B,C), numbers of differentially expressed genes in the treated and control samples compared with the non-treated samples. NoT1, MoT1, BuT1, MoT2, BuT2, MoT3, BuT3 represent the 0-day non-treatment group, the 1-day treatment group, the 1-day control group, the 2-day treatment group, the 2-day control group, the 3-day treatment group, and the 3-day control group, respectively.

Figure S7 | Volcano plots show that the treated rice samples have more differentially expressed genes relative to the non-treated rice samples than the control rice samples. MoT1 vs. NoTO, MoT2 vs. NoTO, and MoT3 vs. NoTO, and MoT3vsNoTO represent the comparisons between the treated rice and the non-treated rice after MoHrip1 treatment for 1, 2, and 3 days. BuT1 vs. NoT0, BuT2 vs. NoTO, and BUT3 vs. NoTO represent the comparisons between the control and non-treated rice groups after treatment with protein buffer for 1, 2, and 3 days.

Figure S8 | Root growth promotion of rice after treated with MoHrip1 or Tris-Cl. (A) Root growth promotion of rice plants. (B) Average length of rice plant root. MM (Rice immersed in MoHrip1 and sprayed with MoHrip1), M (Rice immersed in MoHrip1 and sprayed with Tris-Cl), and CK (Rice immersed in $\mathrm{H}_{2} \mathrm{O}$ and sprayed with Tris-Cl) were measured after 7 days inoculation with $M$. oryzae spores. Values are presented as the mean $\pm \mathrm{SD}$ of three replicates, each consisting of 25 seedlings. Asterisks indicate statistically significant differences, as measured using SAS software (Duncan's $t$ test, $* * p<0.01,{ }^{*} p$ $<0.05)$.

\section{Table S1 | Primer sequences used in this study.}

Table S2 | Statistics of the pathway enrichment of differentially expressed genes detected in treated rice after 1 day. A corrected $p<0.05$ represents statistically significant enrichment of that pathway.

Table S3 | Ct and $\log _{2}$ RPKM values for selected genes. Genes were designated as "undetected" when RPKM $=0.01$

Table S4 | List of the gene IDs and their functional descriptions from two detailed gene clustering analyses.

Table S5 | Eighty genes with particular expression patterns in the treated rice and low or undetected expression in the control rice. Gene IDs with bold fonts were selected for qRT-PCR validation.

Bari, R., and Jones, J. D. G. (2009). Role of plant hormones in plant defence responses. Plant Mol. Biol. 69, 473-488. doi: 10.1007/s11103-008-9435-0

Benjamini, Y., and Hochberg, Y. (1995). Controlling the false discovery rate: a practical and powerful approach to multiple testing. J. R. Stat. Soc. B 57, 289-300.

Bent, A. F., and Mackey, D. (2007). Elicitors, effectors, and r genes: the new paradigm and a lifetime supply of questions. Annu. Rev. Phytopathol. 45, 399-436. doi: 10.1146/annurev.phyto.45.062806.094427

Bowling, S. A., Guo, A., Cao, H., Gordon, A. S., Klessig, D. F., and Dong, X. (1994). A mutation in Arabidopsis that leads to constitutive expression of systemic acquired resistance. Plant Cell 6, 1845-1857. doi: 10.1105/tpc.6.12.1845

Campbell, E. J., Schenk, P. M., Kazan, K., Penninckx, I. A. M. A., Anderson, J. P., Maclean, D. J., et al. (2003). Pathogen-responsive expression of a putative ATP-binding cassette transporter gene conferring resistance to the diterpenoid sclareol is regulated by multiple defense signaling pathways in Arabidopsis. Plant Physiol. 133, 1272-1284. doi: 10.1104/pp.103.024182

Chaman, M. E., Copaja, S. V., and Argandoña, V. H. (2003). Relationships between salicylic acid content, phenylalanine ammonia-lyase (PAL) activity, and resistance of barley to aphid infestation. J. Agricult. Food Chem. 51, 2227-2231. doi: 10.1021/jf020953b 
Chen, M., Zeng, H., Qiu, D., Guo, L., Yang, X., Shi, H., et al. (2012). Purification and characterization of a novel hypersensitive response-inducing elicitor from Magnaporthe oryzae that triggers defense response in rice. PLoS ONE 7:e37654. doi: 10.1371/journal.pone.0037654

Chen, S., Songkumarn, P., Venu, R. C., Gowda, M., Bellizzi, M., Hu, J., et al. (2013). Identification and characterization of in planta-expressed secreted effector proteins from Magnaporthe oryzae that induce cell death in rice. Mol. Plant Microbe Interact. 26, 191-202. doi: 10.1094/MPMI-05-12-0117-R

Chen, X., and Ronald, P. C. (2011). Innate immunity in rice. Trends Plant Sci. 16, 451-459. doi: 10.1016/j.tplants.2011.04.003

Chico, J. M., Chini, A., Fonseca, S., and Solano, R. (2008). JAZ repressors set the rhythm in jasmonate signaling. Curr. Opin. Plant Biol. 11, 486-494. doi: $10.1016 /$ j.pbi.2008.06.003

Chittoor, J. M., Leach, J. E., and White, F. F. (1997). Differential induction of a peroxidase gene family during infection of rice by Xanthomonas oryzae pv. oryzae. Mol. Plant Microbe Interact. 10, 861-871. doi: 10.1094/MPMI.1997.10.7.861

Claeys, H., De Bodt, S., and Inzé, D. (2014). Gibberellins and DELLAs: central nodes in growth regulatory networks. Trends Plant Sci. 19, 231-239. doi: 10.1016/j.tplants.2013.10.001

Daw, B. D., Zhang, L. H., and Wang, Z. Z. (2008). Salicylic acid enhances antifungal resistance to Magnaporthe grisea in rice plants. Aust. Plant Pathol. 37, 637-644. doi: 10.1071/AP08054

Day, R. B., Akimoto-Tomiyama, C., Yazaki, J., Nakamura, K., Fujii, F., Shimbo, K., et al. (2002). Large-scale identification of elicitor-responsive genes in suspension-cultured rice cells by DNA microarray. Plant Biotechnol. 19, 153-155. doi: 10.5511/plantbiotechnology.19.153

De Vleesschauwer, D., Gheysen, G., and Höfte, M. (2013). Hormone defense networking in rice: tales from a different world. Trends Plant Sci. 18, 555-565. doi: 10.1016/j.tplants.2013.07.002

De Wit, P. J. G. M., Mehrabi, R., Van den Burg, H. A., and Stergiopoulos, I. (2009). Fungal effector proteins: past, present and future. Mol. Plant Pathol. 10, 735-747. doi: 10.1111/j.1364-3703.2009.00591.x

Duan, L., Liu, H., Li, X., Xiao, J., and Wang, S. (2014). Multiple phytohormones and phytoalexins are involved in disease resistance to Magnaporthe oryzae invaded from roots in rice. Physiol. Plant. 152, 486-500. doi: 10.1111/ppl.12192

Ellis, B. E., and Amrhein, N. (1971). The 'NIH-Shift' during aromatic ortho-hydroxylation in higher plants. Phytochemistry 10, 3069-3072. doi: 10.1016/S0031-9422(00)97354-0

Ellis, J. G., Rafiqi, M., Gan, P., Chakrabarti, A., and Dodds, P. N. (2009). Recent progress in discovery and functional analysis of effector proteins of fungal and oomycete plant pathogens. Curr. Opin. Plant Biol. 12, 399-405. doi: 10.1016/j.pbi.2009.05.004

Fonseca, S., Chico, J. M., and Solano, R. (2009). The jasmonate pathway: the ligand, the receptor and the core signalling module. Curr. Opin. Plant Biol. 12, 539-547. doi: 10.1016/j.pbi.2009.07.013

Gutterson, N., and Reuber, T. L. (2004). Regulation of disease resistance pathways by AP2/ERF transcription factors. Curr. Opin. Plant Biol. 7, 465-471. doi: 10.1016/j.pbi.2004.04.007

Hansen, K. D., Wu, Z., Irizarry, R. A., and Leek, J. T. (2011). Sequencing technology does not eliminate biological variability. Nat. Biotechnol. 29, 572-573. doi: 10.1038/nbt.1910

Hou, X., Ding, L., and Yu, H. (2013). Crosstalk between GA and JA signaling mediates plant growth and defense. Plant Cell Rep. 32, 1067-1074. doi: 10.1007/s00299-013-1423-4

Huang, J., Yang, X., Wang, M.-M., Tang, H.-J., Ding, L.-Y., Shen, Y., et al. (2007). A novel rice $\mathrm{C}_{2} \mathrm{H}_{2}$-type zinc finger protein lacking DLN-box/EARmotif plays a role in salt tolerance. Biochim. Biophys. Acta 1769, 220-227. doi: 10.1016/j.bbaexp.2007.02.006

Hwang, D. H., Kim, S. T., Kim, S. G., and Kang, K. Y. (2007). Comprehensive analysis of the expression of twenty-seven beta-1, 3-glucanase genes in rice (Oryza sativa L.). Mol. Cells 23, 207-214.

Islam, M. S., Hur, J. H., and Wang, M. H. (2009). The influence of abiotic stresses on expression of zinc finger protein gene in rice. Russ. J. Plant Physiol. 56, 695-701. doi: $10.1134 /$ S1021443709050161

Iwai, T., Seo, S., Mitsuhara, I., and Ohashi, Y. (2007). Probenazole-induced accumulation of salicylic acid confers resistance to Magnaporthe grisea in adult rice plants. Plant Cell Physiol. 48, 915-924. doi: 10.1093/pcp/pcm062
Jasiński, M., Stukkens, Y., Degand, H., Purnelle, B., Marchand-Brynaert, J., and Boutry, M. (2001). A plant plasma membrane ATP binding cassettetype transporter is involved in antifungal terpenoid secretion. Plant Cell 13, 1095-1107. doi: 10.1105/tpc.13.5.1095

Kaneda, T., Taga, Y., Takai, R., Iwano, M., Matsui, H., Takayama, S., et al. (2009). The transcription factor OsNAC4 is a key positive regulator of plant hypersensitive cell death. EMBO J. 28, 926-936. doi: 10.1038/emboj.2009.39

Kanehisa, M., Araki, M., Goto, S., Hattori, M., Hirakawa, M., Itoh, M., et al. (2008). KEGG for linking genomes to life and the environment. Nucleic Acids Res. 36, D480-D484. doi: 10.1093/nar/gkm882

Kawahara, Y., de la Bastide, M., Hamilton, J. P., Kanamori, H., McCombie, W. R., Ouyang, S., et al. (2013). Improvement of the Oryza sativa Nipponbare reference genome using next generation sequence and optical map data. Rice (N.Y.) 6:4. doi: 10.1186/1939-8433-6-4

Kim, C. Y., Lee, S. H., Park, H. C., Bae, C. G., Cheong, Y. H., Choi, Y. J., et al. (2000). Identification of rice blast fungal elicitor-responsive genes by differential display analysis. Mol. Plant Microbe Interact. 13, 470-474. doi: 10.1094/MPMI.2000.13.4.470

Koga, J., Yamauchi, T., Shimura, M., Ogawa, N., Oshima, K., Umemura, K., et al. (1998). Cerebrosides, A., and C, sphingolipid elicitors of hypersensitive cell death and phytoalexin accumulation in rice plants. J. Biol. Chem. 273, 31985-31991. doi: 10.1074/jbc.273.48.31985

Langmead, B., Trapnell, C., Pop, M., and Salzberg, S. L. (2009). Ultrafast and memory-efficient alignment of short DNA sequences to the human genome. Genome Biol. 10, 1-10. doi: 10.1186/gb-2009-10-3-r25

Li, J., Brader, G., and Palva, E. T. (2004). The WRKY70 transcription factor: a node of convergence for jasmonate-mediated and salicylate-mediated signals in plant defense. Plant Cell 16, 319-331. doi: 10.1105/tpc.016980

Liu, W., Liu, J., Ning, Y., Ding, B., Wang, X., Wang, Z., et al. (2013). Recent progress in understanding PAMP- and effector-triggered immunity against the rice blast fungus Magnaporthe oryzae. Mol. Plant 6, 605-620. doi: $10.1093 / \mathrm{mp} / \mathrm{sst} 015$

Maeda, H., and Dudareva, N. (2012). The shikimate pathway and aromatic amino Acid biosynthesis in plants. Annu. Rev. Plant Biol. 63, 73-105. doi: 10.1146/annurev-arplant-042811-105439

Mao, X., Cai, T., Olyarchuk, J. G., and Wei, L. (2005). Automated genome annotation and pathway identification using the KEGG Orthology (KO) as a controlled vocabulary. Bioinformatics 21, 3787-3793. doi: 10.1093/bioinformatics/bti430

Matsuura, H., Aoi, A., Satou, C., Nakaya, M., Masuta, C., and Nabeta, K. (2009). Simultaneous UPLC MS/MS analysis of endogenous jasmonic acid salicylic acid, and their related compounds. Plant Growth Reg. 57, 293-301. doi: 10.1007/s10725-008-9347-7

McGee, J. D., Hamer, J. E., and Hodges, T. K. (2001). Characterization of a PR-10 pathogenesis-related gene family induced in rice during infection with Magnaporthe grisea. Mol. Plant Microbe Interact. 14, 877-886. doi: 10.1094/MPMI.2001.14.7.877

Mentlak, T. A., Kombrink, A., Shinya, T., Ryder, L. S., Otomo, I., Saitoh, H., et al. (2012). Effector-mediated suppression of chitin-triggered immunity by Magnaporthe oryzae is necessary for rice blast disease. Plant Cell 24, 322-335. doi: 10.1105/tpc.111.092957

Metzker, M. L. (2010). Applications of next generation sequencing sequencing technologies - the next generation. Nat. Rev. Genet. 11, 31-46. doi: $10.1038 / \mathrm{nrg} 2626$

Miyamoto, K., Shimizu, T., and Okada, K. (2014). Transcriptional regulation of the biosynthesis of phytoalexin: a lesson from specialized metabolites in rice. Plant Biotechnol. 31, 377-388. doi: 10.5511/plantbiotechnology.14.0730a

Morrissy, A. S., Morin, R. D., Delaney, A., Zeng, T., McDonald, H., Jones, S., et al. (2009). Next-generation tag sequencing for cancer gene expression profiling. Genome Res. 19, 1825-1835. doi: 10.1101/gr.094482.109

Mortazavi, A., Williams, B. A., McCue, K., Schaeffer, L., and Wold, B. (2008). Mapping and quantifying mammalian transcriptomes by RNA-Seq. Nat. Methods 5, 621-628. doi: 10.1038/nmeth.1226

Muthukrishnan, S., Liang, G. H., Trick, H. N., and Gill, B. S. (2001). Pathogenesisrelated proteins and their genes in cereals. Plant Cell Tiss. Org. Cult. 64, 93-114. doi: 10.1023/A:1010763506802

Nomura, T., Ishihara, A., Imaishi, H., Endo, T. R., Ohkawa, H., and Iwamura, H. (2002). Molecular characterization and chromosomal 
localization of cytochrome P450 genes involved in the biosynthesis of cyclic hydroxamic acids in hexaploid wheat. Mol. Genet. Genomics 267, 210-217. doi: 10.1007/s00438-002-0653-x

Nürnberger, T. (1999). Signal perception in plant pathogen defense. Cell. Mol. Life Sci. 55, 167-182. doi: $10.1007 / \mathrm{s} 000180050283$

Park, C.-H., Chen, S., Shirsekar, G., Zhou, B., Khang, C. H., Songkumarn, P., et al. (2012). The Magnaporthe oryzae effector AvrPiz-t targets the RING E3 ubiquitin ligase APIP6 to suppress pathogen-associated molecular pattern-triggered immunity in rice. Plant Cell 24, 4748-4762. doi: 10.1105/tpc.112.105429

Peng, D.-H., Qiu, D.-W., Ruan, L.-F., Zhou, C.-F., and Sun, M. (2011). Protein elicitor PemG1 from Magnaporthe grisea induces systemic acquired resistance (SAR) in plants. Mol. Plant Microbe Interact. 24, 1239-1246. doi: 10.1094/MPMI-01-11-0003

Qiu, D., Mao, J., Yang, X., and Zeng, H. (2009). Expression of an elicitor-encoding gene from Magnaporthe grisea enhances resistance against blast disease in transgenic rice. Plant Cell Rep. 28, 925-933. doi: 10.1007/s00299-009-0698-y

Rakwal, R., Agrawal, G. K., and Agrawal, V. P. (2001). Jasmonate, salicylate, protein phosphatase $2 \mathrm{~A}$ inhibitors and kinetinup-regulate OsPR5 expression in cut-responsive rice (Oryza sativa). J. Plant Physiol. 158, 1357-1362. doi: 10.1078/0176-1617-00616

Rauyaree, P., Choi, W., Fang, E., Blackmon, B., and Dean, R. A. (2001). Genes expressed during early stages of rice infection with the rice blast fungus Magnaporthe grisea. Mol. Plant Pathol. 2, 347-354. doi: 10.1046/j.1464-6722.2001.00085.x

Ribot, C., Hirsch, J., Balzergue, S., Tharreau, D., Nottéghem, J.-L., Lebrun, M.-H., et al. (2008). Susceptibility of rice to the blast fungus, Magnaporthe grisea. J. Plant Physiol. 165, 114-124. doi: 10.1016/j.jplph.2007.06.013

Saitoh, H., Fujisawa, S., Mitsuoka C., Ito, A., Hirabuchi, A., Ikeda, K., et al. (2012). Large-scale gene disruption in Magnaporthe oryzae identifies MC69, a secreted protein required for infection by monocot and dicot fungal pathogens. PLoS Pathog. 8, 657-665. doi: 10.1371/journal.ppat.1002711

Sasaki, T., and Burr, B. (2000). International rice genome sequencing project: the effort to completely sequence the rice genome. Curr. Opin. Plant Biol. 3, 138-141. doi: 10.1016/S1369-5266(99)00047-3

Shimono, M., Sugano, S., Nakayama, A., Jiang, C.-J., Ono, K., Toki, S., et al. (2007). Rice WRKY45 plays a crucial role in benzothiadiazoleinducible blast resistance. Plant Cell 19, 2064-2076. doi: 10.1105/tpc.106. 046250

Singh, K. B., Foley, R. C., and Oñate-Sánchez, L. (2002). Transcription factors in plant defense and stress responses. Curr. Opin. Plant Biol. 5, 430-436. doi: 10.1016/S1369-5266(02)00289-3

Sun, L., Huang, L., Hong, Y., Zhang, H., Song, F., and Li, D. (2015). Comprehensive analysis suggests overlapping expression of rice ONAC transcription factors in abiotic and biotic stress responses. Int. J. Mol. Sci. 16, 4306-4326. doi: $10.3390 /$ ijms 16024306

Talbot, N. J. (2003). On the trail of a cereal killer: exploring the biology of Magnaporthe grisea. Annu. Rev. Microbiol. 57, 177-202. doi: 10.1146/ annurev.micro.57.030502.090957

Tao, X., Gu, Y.-H., Wang, H.-Y., Zheng, W., Li, X., Zhao, C.-W., et al. (2012). Digital gene expression analysis based on integrated de novo transcriptome assembly of sweet potato [Ipomoea batatas (L.) Lam]. PLoS ONE 7:e36234. doi: 10.1371/journal.pone.0036234

Thaler, J. S., Humphrey, P. T., and Whiteman, N. K. (2012). Evolution of jasmonate and salicylate signal crosstalk. Trends Plant Sci. 17, 260-270. doi: 10.1016/j.tplants.2012.02.010
Trapnell, C., Pachter, L., and Salzberg, S. L. (2009). TopHat: discovering splice junctions with RNA-Seq. Bioinformatics 25, 1105-1111. doi: 10.1093/ bioinformatics/btp120

Umemura, K., Ogawa, N., Shimura, M., Koga, J., Usami, H., and Kono, T. (2014). Possible role of phytocassane, rice phytoalexin, in disease resistance of rice against the blast fungusmagnaporthe grisea. Biosci. Biotechnol. Biochem. 67, 899-902. doi: 10.1271/bbb.67.899

Wang, L., Feng, Z., Wang, X., Wang, X., and Zhang, X. (2010). DEGseq: an $\mathrm{R}$ package for identifying differentially expressed genes from RNA-seq data. Bioinformatics 26, 136-138. doi: 10.1093/bioinformatics/btp612

Wang, Q., Hillwig, M. L., Okada, K., Yamazaki, K., Wu, Y., Swaminathan, S., et al. (2012). Characterization of CYP76M5-8 indicates metabolic plasticity within a plant biosynthetic gene cluster. J. Biol. Chem. 287, 6159-6168. doi: 10.1074/jbc.M111.305599

Wei, T., Ou, B., Li, J., Zhao, Y., Guo, D., Zhu, Y., et al. (2013). Transcriptional profiling of rice early response to Magnaporthe oryzae identified OsWRKYs as important regulators in rice blast resistance. PLoS ONE 8:e59720. doi: 10.1371/journal.pone.0059720

Xiao, J., Jin, X., Jia, X., Wang, H., Cao, A., Zhao, W., et al. (2013). Transcriptome-based discovery of pathways and genes related to resistance against Fusarium head blight in wheat landrace Wangshuibai. BMC Genomics 14:197. doi: 10.1186/1471-2164-14-197

Yan, S., and Dong, X. (2014). Perception of the plant immune signal salicylic acid. Curr. Opin. Plant Biol. 20, 64-68. doi: 10.1016/j.pbi.2014.04.006

Yang, D. L., Yao, J., Mei, C. S., Tong, X. H., Zeng, L. J., Li, Q., et al. (2012). Plant hormone jasmonate prioritizes defense over growth by interfering with gibberellin signaling cascade. Proc. Natl. Acad. Sci. U.S.A. 109, E1192-E1200. doi: 10.1073/pnas.1201616109

Young, M. D., Wakefield, M. J., Smyth, G. K., and Oshlack, A. (2010). Gene ontology analysis for RNA-seq: accounting for selection bias. Genome Biol. 11:R14. doi: 10.1186/gb-2010-11-2-r14

Yu, D., Liu, Y., Fan, B., Klessig, D. F., and Chen, Z. (1997). Is the high basal level of salicylic acid important for disease resistance in potato? Plant Physiol. 115, 343-349. doi: 10.1104/pp.115.2.343

Zhang, C., Liu, X., Qiu, D., and Zeng, H. (2013). Purification, crystallization and preliminary X-ray diffraction analysis of the effector protein MoHrip1 from Magnaporthe oryzae. Acta Crystallogr. F. Struct. Biol. Cryst. Commun. 69, 460-462. doi: 10.1107/S1744309113006490

Zhou, N., Tootle, T. L., and Glazebrook, J. (1999). Arabidopsis PAD3, a gene required for camalexin biosynthesis, encodes a putative cytochrome $\mathrm{P} 450$ monooxygenase. Plant Cell 11, 2419-2428. doi: 10.1105/tpc.11.12.2419

Zhu-Salzman, K., Salzman, R. A., Koiwa, H., Murdock, L. L., Bressan, R. A., and Hasegawa, P. M. (1998). Ethylene negatively regulates local expression of plant defense lectin genes. Physiol. Plant. 104, 365-372. doi: 10.1034/j.1399-3054.1998.1040311.x

Conflict of Interest Statement: The authors declare that the research was conducted in the absence of any commercial or financial relationships that could be construed as a potential conflict of interest.

Copyright (๑) 2016 Lv, Wang, Yang, Guo, Qiu and Zeng. This is an open-access article distributed under the terms of the Creative Commons Attribution License (CC BY). The use, distribution or reproduction in other forums is permitted, provided the original author(s) or licensor are credited and that the original publication in this journal is cited, in accordance with accepted academic practice. No use, distribution or reproduction is permitted which does not comply with these terms. 